\title{
Fault Detection Filter Design of Polytopic Uncertain Continuous-Time Singular Markovian Jump Systems with Time-Varying Delays
}

\author{
Yunling Shi $\mathbb{C}^{1,2}$ and Xiuyan Peng $\mathbb{D}^{1}$ \\ ${ }^{1}$ College of Automation, Harbin Engineering University, Harbin 150001, China \\ ${ }^{2}$ Department of Electrical and Information Engineering, Heilongjiang University of Technology, Jixi, Heilongjiang 158100, China
}

Correspondence should be addressed to Xiuyan Peng; pengxiuyan163@163.com

Received 15 October 2019; Revised 22 December 2019; Accepted 8 January 2020; Published 12 February 2020

Academic Editor: Wenqin Wang

Copyright (c) 2020 Yunling Shi and Xiuyan Peng. This is an open access article distributed under the Creative Commons Attribution License, which permits unrestricted use, distribution, and reproduction in any medium, provided the original work is properly cited.

\begin{abstract}
This paper investigates the problem of full-order and reduced-order fault detection filter (FDF) design under unified linear matrix inequality (LMI) conditions for a class of continuous-time singular Markovian jump systems (CTSMJSs) with time-varying delays and polytopic uncertain transition rates. By constructing a new Lyapunov function, sufficient conditions are firstly provided for the singular model error augmented system such that the system is stochastically admissible with an $H_{\infty}$ performance level $\gamma$. And then, by applying a novel convex polyhedron technique to decoupled linear matrix inequalities, the full-order and reduced-order fault detection filter parameters can be obtained within a convex optimization frame. The reduced-order fault detection filter (FDF) can not only meet the fault detection accuracy requirements of complex systems but also improve the fault detection efficiency. Finally, a DC motor and an illustrative simulation example are given to verify the feasibility and effectiveness of the proposed algorithms.
\end{abstract}

\section{Introduction}

Markovian jump systems (MJSs) are usually defined as a family of complex random jumping parameter systems [1-4]. The past few decades have witnessed the prosperous research on MJSs because it can simulate the structure and parameter changes of real dynamic systems, such as biological economics systems, network control systems, power electronic systems, and mechanical engineering systems [5-9]. It is known that singular systems are completely different from ordinary systems, and when dealing with the stability of singular systems, we need to consider its regularity, the unique impulse-free solution, and admissibility, which are not considered in ordinary systems [10-17]. However, the singular systems have attracted much attention, and many studied results about singular systems have been reported because of their extensively practical applications, such as the filtering problem investigated in $[10,11]$,
$H_{\infty}$ control problem studied in [12], stabilization problem addressed in [13], dissipative control problem investigated in [14], dynamic output feedback control problem considered in [15], and sliding mode control presented in [16].

It is worth mentioning that when the structure or parameters of the singular dynamic hybrid system change, the singular Markovian jump systems (SMJSs) can effectively simulate this phenomenon by using the Markov chain transformation, and many fruitful about SMJSs have been shown in recent years [17-19]. The transition rates (TRs) are the concrete expression of Markov chains, which can express the random transition state of the actual dynamic systems [20-22]. As the soul of SMJSs, TRs are generally considered to be completely known, partly unknown, and completely unknown in most studies [23-25]. It is worth noticing that the TRs with the polytopic uncertain cases are often encountered in practical applications, when the switching mode of the system is uncertain. Due to limitation of measurement conditions 
under a harsh environment, component failures, and random abrupt changes of system structures, the system parameters changed randomly such that the RTs may be polytopic uncertain systems [26-28], for example, network control systems, VTOL (vertical take-off and landing machine) systems, and power systems. However, the fault detection filter of polytopic uncertain SMJSs has not been studied so far, which is the first motivation of our study.

In practice, however, the components or instrument are not always normal, and faults are unavoidable under practical conditions. More generally, the faults are often encountered in various forms. In order to improve the safety performance of the control systems, when the systems fail, we need to quickly detect the faults and avoid accidents. In order to pursue higher security and reliability, many scholars have studied the fault detection problems of Markovian jump systems [29-31]. These scholars only studied the fault detection problems of the ordinary Markovian jump systems. Since the singular Markovian jump systems are more complex than the ordinary Markovian jump systems, the stability, regularity, and nonimpulsiveness need to be considered [32-35]; especially for polytopic uncertain continuous-time singular Markovian jump systems, few results can be found related to the fault detection filter designed problems under this case.

Additionally, it has been shown that time-varying delays are universal in real applications because of the signal transmission in communication, and it can lead to systems performance degradation and instability [34-38]. Inevitably, time delays also exist in SMJSs, and it is necessary to investigate the SMJSs with time-varying delays. As a hot topic, many fruits involving time-varying delays of SMJSs fault detection have been addressed [33-35]. It is worth mentioning that the fault detection of polytopic uncertain CTSMJSs with time-varying delays, which contains completely known, partly unknown, and completely unknown TRs, has not been studied. The polytopic uncertain SMJSs with time-varying delays can summarize actual systems more comprehensively, which is the other main motivation of this paper.

From what has been discussed above, in this paper, we will tackle the stochastic admissibility problem for polytopic uncertain CTSMJSs with time-varying delays via constructing a novel Lyapunov function. Then, based on a convex polyhedron technique to decoupled linear matrix inequalities, the full-order and reduced-order fault detection filters can be obtained. In particular, full-order and reduced-order FDF parameters can be computed in a convex optimization frame. On the premise of satisfying the requirement of fault detection accuracy, the proposed reduced-order FDF can effectively reduce the data storage and is less sensitive to external disturbance. The effectiveness of the derived results has been demonstrated by two illustrative examples.

The main contributions and novelties of this paper are summarized as follows. (I) The full-order and reduced-order FDFs for polytopic uncertain CTSMJSs with time-varying delays are proposed and designed for the first time. (II) The innovation reduced-order FDF design method of polytopic uncertain CTSMJSs with time-varying delays is proposed, which satisfies the stochastically admissible sufficient conditions and the $H_{\infty}$ performance standard $\gamma_{\min }$ for the first time. (III) By applying a novel decoupling technique, it has shown the full-order and reduced-order FDFs designed under a unified framework. Therefore, we can get the parameters of the full-order and reduced-order FDFs by adjusting the dimension of the decoupling matrix parameters, which can effectively improve the full-order and reduced-order FDF design scheme. (IV) In this paper, the proposed method not only can calculate parameters of fullorder and reduced-order FDFs as the state TRM is polytopically uncertain but also can obtain the parameters of fullorder and reduced-order FDFs when state TRM is completely under known, partly unknown, and completely unknown circumstances, and their performances are compared on conservative by simulation analysis.

Notations. The notations presented here are standard. $E[\cdot]$ stands for the mathematical expectation. $\operatorname{sym}(K)$ stands for $K+K^{T} . I^{n}$ represents the $n$-dimensional identity matrix, and 0 represents the zero matrix. $R$ is the set of real number, $R^{n}$ denotes the $n$-dimensional Euclidean space, and $R^{m \times n}$ is the set of $m \times n$ real matrix. About real symmetric matrix $P$, $P>0(P \geq 0)$ means that $\mathrm{P}$ is the real symmetric positive (semipositive) and $*$ represents the symmetric term that has been ellipsis. $\|\cdot\|$ denotes the Euclidean norm for vectors. $l_{2}[0, \infty)$ is the space of square summable infinite sequence, and for $w=\{w(t)\} \in l_{2}[0, \infty)$, its norm is given by $\|w\|_{2}=\sqrt{\int_{0}^{\infty}\|w\|^{2} d t}$.

\section{Problem Formulation}

In this section, we consider a class of CTSMJSs with timevarying delays on a complete probability space, which is described as follows:

$$
\left\{\begin{array}{l}
E \dot{x}(t)=A(r(t)) x(t)+A_{d}(r(t)) x(t-d(t))+B(r(t)) u(t) \\
+E(r(t)) w(t)+F(r(t)) f(t), \\
y(t)=C(r(t)) x(t)+C_{d}(r(t)) x(t-d(t))+D(r(t)) w(t) \\
+H(r(t)) f(t),
\end{array}\right.
$$

where $u(t) \in R^{n_{u}}$ represents the known input; $x(t) \in R^{n_{x}}$ stands for the plant state; $w(t) \in R^{n_{d}}$ is the exogenous disturbance signal; and $f(t) \in R^{n_{f}}$ is the fault signal to be detected. $E \in R^{n \times n}$ with $\operatorname{rank}(E)=r \leq n_{x} ; u(t), w(t)$, and $f(t)$, respectively, belong to $l_{2}[0, \infty)$. In addition, $d(t)$ denotes the time-varying delay and satisfying $0 \leq d_{1} \leq d(t) \leq d_{2}<\infty, \dot{d}(t) \leq u<\infty$, for all $t \geq 0$, where $d_{1}$, $d_{2}$, and $u$ are scalars, $\left\{d_{1}, d_{2}\right\} \in R_{+}$represents the lower and upper delay bounds, respectively. $\{r(t), t \geq 0\}$ is a rightcontinuous Markov process, which takes values in a finite state-space set $S=\{1,2, \ldots, N\}$ with the mode transition rate matrix (TRM) $\Pi \triangleq\left\{\lambda_{i j}\right\},(i, j \in S)$, given by

$$
\left.P_{r}\{r(t+\Delta)\}=j \mid r(t)=i\right\}=\left\{\begin{array}{l}
\lambda_{i j} \Delta+0(\Delta), \quad i \neq j, \\
1+\lambda_{i i} \Delta+0(\Delta), \quad i=j,
\end{array}\right.
$$

where $\Delta>0, \lim _{\Delta \longrightarrow 0}(0(\Delta) / \Delta)=0$, and $\lambda_{i j} \geq 0$ is the transition rate from mode $i$ at time $t$ to mode $j$ at time $t+\Delta$ for $i \neq j$. 
For convenience, let $r(t)=i$, such that real known constant system matrices $\left\{A(r(t)), A_{d}(r(t)), B(r(t)), C(r(t)), C_{d}\right.$ $(r(t)), D(r(t)), E(r(t)), F(r(t)), H(r(t))\}$ can be written as $\left\{A_{i}, A_{d i}, B_{i}, C_{i}, C_{d i}, D_{i}, E_{i}, F_{i}, H_{i}\right\}$. The TRs of the Markovian jump process are considered to be partly accessible and polytopic uncertain; that is, the TRM $\Lambda=\left[\lambda_{i j}\right]_{n \times n}$ is hypothesized to belong to a known polytope $P_{\Lambda}$ with vertices $\Lambda_{s}$ :

$$
P_{\Lambda}:=\left\{\Lambda=\sum_{S=1}^{M} \alpha_{s} \Lambda_{s} ; \alpha_{s} \geq 0, \sum_{S=1}^{M} \alpha_{S}=1\right\},
$$

where $\Lambda_{s}=\left[\lambda_{i j}\right]_{n \times n}, i, j \in I, S=1,2, \ldots, M$ are known given TRMs containing known, unknown, and polytopic uncertain elements still. For instance, the TRM of system (1) with three operation modes may be expressed as

$$
\left[\begin{array}{llll}
\lambda_{11} & \tilde{\lambda}_{12} & \tilde{\lambda}_{13} & \lambda_{14} \\
\hat{\lambda}_{21} & \lambda_{22} & \hat{\lambda}_{23} & \lambda_{24} \\
\lambda_{31} & \lambda_{32} & \hat{\lambda}_{33} & \hat{\lambda}_{34} \\
\lambda_{41} & \tilde{\lambda}_{42} & \hat{\lambda}_{43} & \lambda_{44}
\end{array}\right],
$$

where unknown and polytopic uncertain TRs are tagged with hat " "» and " " , respectively, and the others are known as TRs. For notational clarity, we describe $S=S_{k}^{(i)} \cup S_{u k}^{(i)} \cup S_{u c}^{(i)}$ as follows:

$$
\begin{aligned}
& S_{k}^{(i)}:=\left\{j: \lambda_{i j} \text { is known }\right\}, \\
& S_{u k}^{(i)}:=\left\{j: \widehat{\lambda}_{i j} \text { is unknown }\right\}, \\
& S_{u c}^{(i)}:=\left\{j: \widetilde{\lambda}_{i j} \text { is uncertain }\right\} .
\end{aligned}
$$

Also, we can denote

$$
\lambda_{u k}^{(i l)}:=\sum_{j \in S_{u k}^{(i)}} \hat{\lambda}_{i j}=1-\sum_{j \in S_{k}^{(i)}} \lambda_{i j}-\sum_{j \in S_{u c}^{(i)}} \tilde{\lambda}_{i j}^{(l)} .
$$

Remark 1. We define $\lambda_{k}^{(i)}:=\sum_{j \in S^{(i)}} \lambda_{i j}$ and $\lambda_{u c}^{(i l)}:=\sum_{j \in S_{u c}^{(i)}} \tilde{\lambda}_{i j}^{(l)}$. For achievement, the numerical solvability of the performance analysis, when $\widehat{\lambda}_{i i}$ is unknown, by providing a lower bound element $\lambda_{b}^{(i)} \leq-\left(\lambda_{k}^{(i)}+\lambda_{u c}^{(i l)}\right)$ is provided to restrict the diagonal element of TRM. In this paper, our primary mission is to design FDF for original system (1), and the FDF is considered as follows:

$$
\left\{\begin{array}{l}
\dot{x}_{F}(t)=A_{F i} x_{F}(t)+B_{F i} y(t), \\
r_{F}(t)=C_{F i} x_{F}(t)+D_{F i} y(t),
\end{array}\right.
$$

where $r_{F}(t) \in R^{n_{r}}$ is the residual, $x_{F}(t) \in R^{n_{f}}\left(1 \leq n_{f} \leq n_{x}\right)$ is the state estimation of filter, and the full-order and reducedorder FDF parameter matrices $A_{F i} \in R^{n_{f} \times n_{f}}, B_{F i} \in R^{n_{f} \times n_{y}}$, $C_{F i} \in R^{n_{r} \times n_{f}}$, and $D_{F i} \in R^{n_{r} \times n_{y}}$ can be computed, respectively, when $1 \leq n_{f}=n_{x}$ or $1 \leq n_{f}<n_{x}$.

In this paper, we introduce $\bar{f}(s)=W_{f}(s) f(s)$ to improve the performance of the fault detection system by limiting the frequency interval, where $f(s)$ represents the weighting fault signal and $W_{f}(s)$ represents the stable weighting matrix function. $f(s)$ and $\bar{f}(s)$ denote Laplace transforms of $\bar{f}(t)$ and $f(t)$, separately. The minimal statespace realization of $\bar{f}(s)=W_{f}(s) f(s)$ is supposed to be

$$
\left\{\begin{array}{l}
\dot{x}_{f}(t)=A_{w f i} x_{f}(t)+B_{w f i} f(t), \\
\bar{f}(t)=C_{w f i} x_{f}(t)+D_{w f i} f(t),
\end{array}\right.
$$

where $x_{f}(t) \in R^{n_{w}}$ is the state vector and $A_{w f i}, B_{w f i}, C_{w f i}$, and $D_{w f i}$ are arbitrary constant matrices. Notice that $\mathrm{e}(t):=r_{F}(t)-\bar{f}(t)$ denotes the weighted fault estimation error. Considering expressions (1), (7), and (8), the following singular model error augmented system can be obtained:

$$
\left\{\begin{array}{l}
\widetilde{E} \dot{\tilde{x}}(t)=\widetilde{A}_{i} \tilde{x}(t)+\widetilde{A}_{d i} \Phi \widetilde{x}(t-d(t))+\widetilde{B}_{i} \Psi(t), \\
e(t)=\widetilde{C}_{i} \widetilde{x}(t)+\widetilde{C}_{d i} \Phi \widetilde{x}(t-d(t))+\widetilde{D}_{i} \Psi(t),
\end{array}\right.
$$

where $\quad \Psi(t)=\left[\begin{array}{llll}u^{T}(t) & w^{T}(t) & f^{T}(t)\end{array}\right]^{T}, \quad \tilde{x}(t):=$ $\left[\begin{array}{lll}x^{T}(t) & x_{F}^{T}(t) & x_{f}^{T}(t)\end{array}\right]^{T}$, and

$$
\widetilde{E}=\left[\begin{array}{ccc}
E & 0 & 0 \\
0 & I & 0 \\
0 & 0 & I
\end{array}\right],
$$$$
\widetilde{A}_{i}=\left[\begin{array}{ccc}
A_{i} & 0 & 0 \\
B_{F i} C_{i} & A_{F i} & 0 \\
0 & 0 & A_{w f i}
\end{array}\right] \text {, }
$$$$
\widetilde{A}_{d i}=\left[\begin{array}{c}
A_{d i} \\
B_{F i} C_{d i} \\
0
\end{array}\right] \text {, }
$$

$$
\begin{aligned}
\widetilde{B}_{i} & =\left[\begin{array}{ccc}
B_{i} & E_{i} & F_{i} \\
0 & B_{F i} D_{i} & B_{F i} H_{i} \\
0 & 0 & B_{w f i}
\end{array}\right], \\
\widetilde{C}_{i} & =\left[\begin{array}{lll}
D_{F i} C_{i} & C_{F i} & -C_{w f i}
\end{array}\right], \\
\widetilde{C}_{d i} & =\left[\begin{array}{ll}
D_{F i} C_{d i}
\end{array}\right], \\
\widetilde{D}_{i} & =\left[\begin{array}{lll}
0 & D_{F i} D_{i} & D_{F i} H_{i}-D_{w f i}
\end{array}\right], \\
\Phi & =\left[\begin{array}{lll}
I & 0 & 0
\end{array}\right] .
\end{aligned}
$$

Remark 2. $u(t)$ is the known input of the system, which is combined with the disturbance $w(t)$ and fault $f(t)$ to form the input of the singular model error augmented system (9) because when $u(t)=0$, the $H_{\infty}$ performance $\gamma$ and conservativeness of the system will decrease, and it can be regarded as the negative input of the system.

Remark 3. In fact, the singular model error augmented system (9) has the characteristics of singular system. In order to better study the stability of system (9), we must ensure the solution is reasonable for the singular model error augmented system (9) when the input $\Psi(t)=0$. Therefore, we give the following Assumption 1 and Lemma 1.

Assumption 1. For every $i \in I$, there exists a pair of nonsingular matrices $M, N \in R^{\left(n_{x}+n_{f}+n_{w}\right) \times\left(n_{x}+n_{f}+n_{w}\right)}$ such that 


$$
\begin{aligned}
M \widetilde{E} N & =\left[\begin{array}{cc}
I_{\left(r+n_{f}+n_{w}\right)} & 0_{\left(r+n_{f}+n_{w}\right) \times\left(n_{x}-r\right)} \\
0 & 0_{\left(n_{x}-r\right) \times\left(r+n_{f}+n_{w}\right) \times\left(n_{x}-r\right)}
\end{array}\right], \\
M \widetilde{A}_{i} N & =\left[\begin{array}{cc}
\widetilde{A}_{i 1} & \widetilde{A}_{i 2} \\
\widetilde{A}_{i 3} & \widetilde{A}_{i 4}
\end{array}\right], \\
M \widetilde{A}_{d i} N & =\left[\begin{array}{cc}
\widetilde{A}_{d i 1} & \widetilde{A}_{d i 2} \\
\widetilde{A}_{d i 3} & \widetilde{A}_{d i 4}
\end{array}\right] .
\end{aligned}
$$

Lemma 1. Under Assumption 1, the unforced singular model error augmented system (9) has a unique (right-continuous) solution.

Proof. Let $\quad \varsigma(t)=N^{-1} \tilde{x}(t)=\left[\varsigma_{1}^{T}(t) \varsigma_{2}^{T}(t)\right], \quad \varsigma_{1}(t) \epsilon$ $R^{\left(r+n_{f}+n_{w}\right)}$, and $\varsigma_{2}(t) \in R^{\left(n_{x}-r\right)}$, then unforced subsystem (9) can be written as the following form:

$$
\begin{aligned}
& \left\{\begin{array}{l}
\dot{\zeta}_{1}(t)=\widetilde{A}_{i 1} \varsigma_{1}(t)+\widetilde{A}_{i 2} \varsigma_{2}(t)+\widetilde{A}_{d i 1} \varsigma_{1}(t-d(t)) \\
+\widetilde{A}_{d i 1} \varsigma_{2}(t-d(t)), \\
0=\widetilde{A}_{i 3} \varsigma_{1}(t)+\widetilde{A}_{i 4} \varsigma_{2}(t)+\widetilde{A}_{d i 3} \varsigma_{1}(t-d(t)) \\
+\widetilde{A}_{d i 4} \varsigma_{2}(t-d(t)),
\end{array}\right. \\
& \text { or }\left\{\begin{array}{l}
\dot{\varsigma}_{1}(t)=\widetilde{A}_{i 1} \varsigma_{1}(t)+\widetilde{A}_{d i 1} \varsigma_{1}(t-d(t)), \\
0=\widetilde{A}_{i 3} \varsigma_{1}(t)++\widetilde{A}_{d i 3} \varsigma_{1}(t-d(t)), \\
\varsigma_{2}(t)=0 .
\end{array}\right.
\end{aligned}
$$

Therefore, according to [35-38], the unforced input system (9) has a unique impulse-free solution for all $t \geq 0$ when Assumption 1 holds. Before proceeding to the next step, we introduce the following important definitions to drive our main results.

Definition 1 (see [11]). The model error augmented system (9) is said to be as follows:

(1) Regular, if any $i \in I$ and $\Psi(t)=0, \operatorname{det}\left(s \widetilde{E}-\widetilde{A}_{i}\right) \neq 0$.

(2) Impulse-free, if any $i \in I$ and $\Psi(t)=0$, $\operatorname{deg}\left(\operatorname{det}\left(s \widetilde{E}-\widetilde{A}_{i}\right)\right)=\operatorname{rank}(\widetilde{E})$.

(3) Stochastically stable, if for any initial state $\tilde{x}(0) \in R^{n_{x}}$, $r(0) \in I$, there exists a scalar $M\left(\tilde{x}_{0}, r_{0}\right)>0$, such that the following inequality holds:

$$
E\left\{\int_{0}^{\infty}\|\widetilde{x}(t)\|^{2} \mid\left(\tilde{x}_{0}, r_{0}\right)\right\} \leq M\left(\tilde{x}_{0}, r_{0}\right) \text {. }
$$

(4) Stochastically admissible, if system (9) is regular, impulse-free, and stochastically stable.

Definition 2 (see [11]). Given a scalar $\gamma>0$ and under the condition $\Psi(t) \in l_{2}[0, \infty)$, singular model error augmented system (9) is said to be stochastically admissible and has an $H_{\infty}$ noise attenuation performance index $\gamma$, if the following conditions can be satisfied.
(1) When $\Psi(t)=0, t \geq 0$, and condition (3) of Definition 1 is satisfied, system (9) is stochastically stable.

(2) When $\Psi(t) \neq 0, t \geq 0$, under zero initial conditions, the following inequality holds:

$$
E\left\{\int_{0}^{\infty} e^{T}(t) e(t) d t\right\}<\gamma^{2} E\left\{\int_{0}^{\infty} \Psi^{T}(t) \Psi(t) d t\right\}
$$

We assume that polytopic uncertain CTSMJSs with time-varying delays (1) is stochastic admissible. The main purpose of this paper is to obtain the parameters of the fault detection filtering system (7) under the premise that the model error augmented system (9) is stochastically admissible with the $H_{\infty}$ performance level $\gamma$. To improve fault detection performance, we define the following residual estimation function $J(\widehat{r}(t))$ and threshold $J_{t h}$ :

$$
\begin{aligned}
J(\widehat{r}(t)) & =\left\{\int_{k_{0}}^{k_{0}+L} \widehat{r}^{T}(t) \widehat{r}(t)\right\}^{1 / 2}, \\
J_{t h} & =\sup _{0 \neq w \in l_{2}, 0 \neq u_{t} \in l_{2}, f=0} E[J(\widehat{r}(t))],
\end{aligned}
$$

where $k_{0}$ represents the moment of initial evaluation and $L$ represents the evaluation time step. If fault $f(t)$ has occurred, it can be detected by the following logical relationships $J(\widehat{r}(t))>J_{t h} \Longrightarrow$ fault happened $\Longrightarrow$ alarm and $J(\widehat{r}(t)) \leq J_{t h} \Longrightarrow$ fault free.

In order to facilitate subsequent research, we will give the following Jensen inequality.

Lemma 2 (see [39]). For any positive-definite symmetric matrix $M \in R^{n \times n}$, scalar $b \geq a$, and vector function: $v:[a, b] \longrightarrow R^{n}$, such that the following inequality holds:

$$
\left(\int_{a}^{b} v(s) d s\right)^{T} M\left(\int_{a}^{b} v(s) d s\right) \leq(b-a) \int_{a}^{b} v^{T}(s) M v(s) d s
$$

\section{Performance Analysis of FDFS}

In this section, the $H_{\infty}$ performance analysis level is firstly derived for system (9). Then, present the FDF design method for CTSMJSs with completely known TRs and time-varying delays.

Theorem 1. Under Assumption 1, given scalars $d_{1}$, $d_{2}\left(0 \leq d_{1} \leq d_{2}\right), u \geq 0$, for any time-varying delays $d(t)$, the continuous-time singular error augmented system (9) is stochastically admissible with an $H_{\infty}$ performance $\gamma$. If there exists symmetric positive-definite matrices $P_{i} \in R^{\left(n_{x}+n_{f}+n_{w}\right) \times\left(n_{x}+n_{f}+n_{w}\right)}, \quad\left\{Z_{1}, Z_{2}, R_{1}, R_{2}, Q_{1 i}, Q_{2 i}, Q_{3 i}\right\} \in$ $R^{n_{x} \times n_{x}}$, appropriate dimensions matrices $\tilde{N}_{i}, \widehat{S} \in$ $R^{\left(n_{x}+n_{f}+n_{w}\right) \times\left(n_{x}-r\right)}$, and satisfying $\widetilde{E}^{T} \widetilde{S}=0$, such that the following LMIs hold for any $i, j \in I$ : 


$$
\begin{aligned}
& \left\{\begin{array}{l}
\sum_{j=1}^{N} \lambda_{i j}\left(Q_{1 j}+Q_{3 j}\right) \leq Z_{2} \\
\sum_{j=1}^{N} \lambda_{i j} Q_{2 j} \leq Z_{1} \\
\sum_{j=1}^{N} \lambda_{i j} Q_{3 j} \leq Z_{2}
\end{array}\right. \\
& \Omega_{i}=\left[\begin{array}{ccccccc}
\Omega_{11} & \Omega_{12} & \widetilde{E}^{T} \Phi^{T} R_{1} \Phi \widetilde{E} & 0 & \Omega_{15} & \Omega_{16} & \widetilde{C}_{i}^{T} \\
* & \Omega_{22} & \widetilde{E}^{T} \Phi^{T} R_{2} \Phi \widetilde{E} & \widetilde{E}^{T} \Phi^{T} R_{2} \Phi \widetilde{E} & 0 & \Omega_{26} & \Phi^{T} \widetilde{C}_{d i}^{T} \\
* & * & \Omega_{33} & 0 & 0 & 0 & 0 \\
* & * & * & \Omega_{44} & 0 & 0 & 0 \\
* & * & * & * & -\gamma^{2} I & \Omega_{56} & \widetilde{D}_{i}^{T} \\
* & * & * & * & * & \Omega_{66} & 0 \\
* & * & * & * & * & * & -I
\end{array}\right]<0, \\
& \Omega_{11}=\sum_{j=1}^{N} \lambda_{i j} \widetilde{E}^{T} P_{j} \widetilde{E}+\operatorname{sym}\left(\widetilde{E}^{T} P_{i} \widetilde{A}_{i}\right)+\operatorname{sym}\left(\widetilde{A}_{i}^{T} \widetilde{S}^{T}{ }_{i}^{T}\right) \\
& +\Phi^{T}\left(Q_{1 i}+Q_{2 i}+Q_{3 i}+d_{1} Z_{1}+d_{2} Z_{2}\right) \Phi-\widetilde{E}^{T} \Phi^{T} R_{1} \Phi \widetilde{E} \\
& \Omega_{12}=\widetilde{E}^{T} P_{i} \widetilde{A}_{d i} \Phi+\widetilde{N}_{i} \widetilde{S}^{T} \widetilde{A}_{d i} \Phi, \\
& \Omega_{15}=\widetilde{N}_{i} \widetilde{S}^{T} \widetilde{B}_{i}+\widetilde{E}^{T} P_{i} \widetilde{B}_{i} \text {, } \\
& \Omega_{16}=\widetilde{A}_{i}^{T} \Phi^{T}\left(d_{1}^{2} R_{1}+d_{12}^{2} R_{2}\right) \Phi, \\
& \left.\Omega_{22}=-(1-u) \Phi^{T} Q_{1 i} \Phi-2 E^{T} \Phi^{T} R_{2} \Phi E\right) \text {, } \\
& \Omega_{26}=\Phi^{T} \widetilde{A}_{d i}^{T} \Phi^{T}\left(d_{1}^{2} R_{1}+d_{12}^{2} R_{2}\right) \Phi, \\
& \Omega_{33}=-\Phi^{T} Q_{2 i} \Phi-E^{T} \Phi^{T}\left(R_{1}+R_{2}\right) \Phi E, \\
& \Omega_{44}=-\Phi^{T} Q_{3 i} \Phi-E^{T} \Phi^{T} R_{2} \Phi E, \\
& \Omega_{56}=\widetilde{B}_{i}^{T} \Phi^{T}\left(d_{1}^{2} R_{1}+d_{12}^{2} R_{2}\right) \Phi, \\
& \Omega_{66}=-\Phi^{T}\left(d_{1}^{2} R_{1}+d_{12}^{2} R_{2}\right) \Phi \text {. }
\end{aligned}
$$

Proof. Firstly, the regularity and impulse-free for system (9) are proved. From (18), we have

$$
\operatorname{sym}\left(\widetilde{E}^{T} P_{i} \widetilde{A}_{i}\right)+\operatorname{sym}\left(\widetilde{A}_{i}^{T} \widetilde{S} \widetilde{N}_{i}^{T}\right)<0,
$$

Suppose that Assumption 1 is satisfied, $\operatorname{rank}(\widetilde{E})=r+$ $n_{f}+n_{w}<n_{x}+n_{f}+n_{w}$ and nonsingular matrices $M, N \in R^{\left(n_{x}+n_{f}+n_{w}\right) \times\left(n_{x}+n_{f}+n_{w}\right)}$ such that

$$
\begin{aligned}
M^{-T} P_{i} M^{-1} & =\left[\begin{array}{ll}
P_{i 1} & P_{i 2} \\
P_{i 3} & P_{i 4}
\end{array}\right], \\
N^{T} \widetilde{N}_{i} & =\left[\begin{array}{c}
\widetilde{N}_{i 1} \\
\widetilde{N}_{i 2}
\end{array}\right], \\
M^{-T} \widetilde{S} & =\left[\begin{array}{l}
0 \\
S
\end{array}\right],
\end{aligned}
$$

where $S \in R^{\left(n_{x}+n_{f}+n_{w}-r\right) \times\left(n_{x}+n_{f}+n_{w}-r\right)}$; premultiplying and postmultiplying (19) by $N^{T}$ and $N$, respectively, we get $\widetilde{A}_{i 4}^{T} S \widetilde{N}_{i 2}^{T}+\widetilde{N}_{i 2} S^{T} \widetilde{A}_{i 4}<0$, and it is easy to see that $\widetilde{A}_{i 4}$ is the nonsingular matrix. According to Lemma 1 and (1) and (2) in Definition 1, the unforced singular model error augmented system (9) is regular and impulse-free. For the stochastic admissible and $H_{\infty}$ property analysis of system (9), under the zero initial condition and suppose $d(t)$ is differentiable, that is, $\Psi(t)=0$, let us define $\tilde{x}_{t}(s)=\tilde{x}(t+s), s \in\left[d_{1}, d_{2}\right]$, and choose the following Lyapunov functional:

$$
V(\tilde{x}(t), i, t)=\sum_{s=1}^{4} V_{s}(\tilde{x}(t), i, t),
$$

where 


$$
\begin{aligned}
& V_{1}(\widetilde{x}(t), i, t)=\widetilde{x}^{T}(t) \widetilde{E}^{T} P_{i} \widetilde{E} \tilde{x}(t) \\
& V_{2}(\tilde{x}(t), i, t)=\int_{t-d(t)}^{t} \tilde{x}^{T}(\alpha) \Phi^{T} Q_{1 i} \Phi \tilde{x}(\alpha) d \alpha+\int_{t-d_{1}}^{t} \tilde{x}^{T}(\alpha) \Phi^{T} Q_{2 i} \Phi \tilde{x}(\alpha) d \alpha+\int_{t-d_{2}}^{t} \tilde{x}^{T}(\alpha) \Phi^{T} Q_{3 i} \Phi \tilde{x}(\alpha) d \alpha \\
& V_{3}(\tilde{x}(t), i, t)=\int_{-d_{1}}^{0} \int_{t+s}^{t} \tilde{x}^{T}(\alpha) \Phi^{T} Z_{1} \Phi \tilde{x}(\alpha) d \alpha d s+\int_{-d_{2}}^{0} \int_{t+s}^{t} \tilde{x}^{T}(\alpha) \Phi^{T} Z_{2} \Phi \tilde{x}(\alpha) d \alpha d s, \\
& V_{4}(\widetilde{x}(t), i, t)=d_{1} \int_{-d_{1}}^{0} \int_{t+s}^{t} \dot{\tilde{x}}^{T}(s) \widetilde{E}^{T} \Phi^{T} R_{1} \Phi \widetilde{E} \dot{\tilde{x}}(s) d \alpha d s+d_{12} \int_{-d_{2}}^{-d_{1}} \int_{t+s}^{t} \dot{\tilde{x}}^{T}(s) \widetilde{E}^{T} \Phi^{T} R_{2} \Phi \widetilde{E} \dot{\tilde{x}}(s) d \alpha d s,
\end{aligned}
$$

where parameter-dependent Lyapunov matrices $P_{i}=P_{i}^{T}$, $Q_{l}=Q_{l}^{T}>0, l \in\{1,2,3\}, Z_{1}=Z_{1}^{T}>0, Z_{2}=Z_{2}^{T}>0$ will be determined. Let $\delta$ be the weak infinitesimal generator of random process $\{\tilde{x}(t), r(t)\}$, for each $r(t)=i, i \in S$, then the infinitesimal operator along the state trajectory of system (9) can be obtained:

$\delta V_{1}(\tilde{x}(t), i, t)$

$$
\begin{aligned}
& =\lim _{\Delta \longrightarrow 0^{+}} \frac{1}{\Delta}\left[\sum_{j=1, j \neq i}^{N} \operatorname{Pr}\{r(t+\Delta) \mid r(t)=i\} \widetilde{x}^{T}(t+\Delta) \widetilde{E}^{T} P_{j} \widetilde{E} \widetilde{x}(t+\Delta)+\operatorname{Pr}\{r(t+\Delta)=i \mid r(t)=i\} \widetilde{x}^{T}(t+\Delta) \times \widetilde{E}^{T} P_{i} \widetilde{E} \widetilde{x}(t+\Delta)\right. \\
& \left.-\widetilde{x}^{T}(t) \widetilde{E}^{T} P_{i} \widetilde{E} \widetilde{x}(t)\right] \\
& =\lim _{\Delta \longrightarrow 0^{+}} \frac{1}{\Delta}\left[\sum_{j=1, j \neq i}^{N}\left[\lambda_{i j} \Delta+0(\Delta)\right][\tilde{x}(t)+\Delta \dot{\tilde{x}}(t)+0(\Delta)]^{T} \widetilde{E}^{T} P_{j} \widetilde{E}[\widetilde{x}(t)+\Delta \dot{\tilde{x}}(t)+0(\Delta)]\right. \\
& \left.+\left(1+\lambda_{i i} \Delta+0(\Delta)\right)[\widetilde{x}(t)+\Delta \dot{\tilde{x}}(t)+0(\Delta)]^{T} \widetilde{E}^{T} P_{j} \widetilde{E}[\widetilde{x}(t)+\Delta \dot{\tilde{x}}(t)+0(\Delta)]-\widetilde{x}^{T}(t) \widetilde{E}^{T} P_{i} \widetilde{E} \widetilde{x}(t)\right] \\
& =\widetilde{x}^{T}(t)\left(\sum_{j=1}^{N} \lambda_{i j} \widetilde{E}^{T} P_{j} \widetilde{E}\right) \tilde{x}(t)+\widetilde{x}^{T}(t) \widetilde{E}^{T} P_{i} \widetilde{E} \dot{\tilde{x}}(t)+\dot{\tilde{x}}^{T}(t) \widetilde{E}^{T} P_{i}^{T} \widetilde{E} \widetilde{x}(t) \\
& =\widetilde{x}^{T}(t)\left(\sum_{j=1}^{N} \lambda_{i j} \widetilde{E}^{T} P_{j} \widetilde{E}\right) \widetilde{x}(t)+\widetilde{x}^{T}(t) \widetilde{E}^{T} P_{i}\left[\widetilde{A}_{i} \tilde{x}(t)+\widetilde{A}_{d i} \Phi \widetilde{x}(t-d(t))\right]+\left[\widetilde{A}_{i} \widetilde{x}(t)+\widetilde{A}_{d i} \Phi \widetilde{x}(t-d(t))\right]^{T} P_{i}^{T} \widetilde{E} \widetilde{x}(t), \\
& \delta V_{2}(\tilde{x}(t), i, t) \\
& =\widetilde{x}^{T}(t) \Phi^{T}\left(Q_{1 i}+Q_{2 i}+Q_{3 i}\right) \Phi \tilde{x}(t)-(1-u) \tilde{x}^{T}(t-d(t)) \Phi^{T} Q_{1 i} \widetilde{E} \widetilde{x}(t-d(t))-\tilde{x}^{T}\left(t-d_{1}\right) \Phi^{T} Q_{2 i} \widetilde{E} \tilde{x}\left(t-d_{1}\right) \\
& -\tilde{x}^{T}\left(t-d_{2}\right) \Phi^{T} Q_{3 i} \widetilde{E} \tilde{x}\left(t-d_{2}\right) \\
& \leq \tilde{x}^{T}(t) \Phi^{T}\left(Q_{1 i}+Q_{2 i}+Q_{3 i}\right) \Phi \widetilde{x}(t)-(1-u) \tilde{x}^{T}(t-d(t)) \Phi^{T} Q_{1 i} \widetilde{E} \widetilde{x}(t-d(t))-\widetilde{x}^{T}\left(t-d_{1}\right) \Phi^{T} Q_{2 i} \widetilde{E} \widetilde{x}\left(t-d_{1}\right) \\
& -\tilde{x}^{T}\left(t-d_{2}\right) \Phi^{T} Q_{3 i} \widetilde{E} \tilde{x}\left(t-d_{2}\right)+\int_{t-d(t)}^{t} \tilde{x}^{T}(\alpha) \Phi^{T}\left(\sum_{j=1}^{N} \lambda_{i j} Q_{1 j}\right) \Phi \tilde{x}(\alpha) d \alpha+\int_{t-d_{1}}^{t} \tilde{x}^{T}(\alpha) \Phi^{T}\left(\sum_{j=1}^{N} \lambda_{i j} Q_{2 j}\right) \Phi \tilde{x}(\alpha) d \alpha \\
& +\int_{t-d_{2}}^{t-d(t)} \tilde{x}^{T}(\alpha) \Phi^{T}\left(\sum_{j=1}^{N} \lambda_{i j} Q_{3 j}\right) \Phi \tilde{x}(\alpha) d \alpha+\int_{t-d(t)}^{t} \tilde{x}^{T}(\alpha) \Phi^{T}\left(\sum_{j=1}^{N} \lambda_{i j} Q_{3 j}\right) \Phi \tilde{x}(\alpha) d \alpha,
\end{aligned}
$$

$\delta V_{3}(\tilde{x}(t), i, t)$

$=\tilde{x}^{T}(t) \Phi^{T}\left[d_{1} Z_{1}+d_{2} Z_{2}\right] \Phi \tilde{x}(t)-\int_{t-d_{1}}^{t} \tilde{x}^{T}(\alpha) \Phi^{T} Z_{1} \Phi \tilde{x}(\alpha) d \alpha-\int_{t-d_{2}}^{t} \tilde{x}^{T}(\alpha) \Phi^{T} Z_{2} \Phi \tilde{x}(\alpha) d \alpha$,

$\delta V_{4}(\tilde{x}(t), i, t)$

$=d_{1}^{2} \dot{\tilde{x}}^{T}(t) \widetilde{E}^{T} \Phi^{T} R_{1} \Phi \widetilde{E} \dot{\tilde{x}}(t)-d_{1} \int_{t-d_{1}}^{t} \dot{\tilde{x}}^{T}(\alpha) \widetilde{E}^{T} \Phi^{T} R_{1} \Phi \widetilde{E} \dot{\tilde{x}}(\alpha) d \alpha+d_{12}^{2} \dot{\tilde{x}}^{T}(t) \widetilde{E}^{T} \Phi^{T} R_{1} \Phi \widetilde{E} \dot{\tilde{x}}(t)$

$-d_{12} \int_{t-d_{2}}^{t-d_{1}} \dot{\tilde{x}}^{T}(\alpha) \widetilde{E}^{T} \Phi^{T} R_{2} \Phi \widetilde{E} \dot{\tilde{x}}(\alpha) d \alpha$. 
According to Lemma 1, we can obtain

$$
\begin{aligned}
-d_{1} \int_{t-d_{1}}^{t} \dot{\tilde{x}}^{T}(\alpha) \widetilde{E}^{T} \Phi^{T} R_{1} \Phi \widetilde{E} \dot{\tilde{x}}(\alpha) d \alpha \leq & \left.\left.-\left(\int_{t-d_{1}}^{t} \Phi \widetilde{E} \dot{\tilde{x}}(\alpha) d \alpha\right)\right)^{T} R_{1}\left(\int_{t-d_{1}}^{t} \Phi \widetilde{E} \dot{\tilde{x}}(\alpha) d \alpha\right)\right) \\
= & -\left[\widetilde{x}(t)-\widetilde{x}\left(t-d_{1}\right)\right]^{T} \widetilde{E}^{T} \Phi^{T} R_{1} \Phi \widetilde{E}\left[\widetilde{x}(t)-\widetilde{x}\left(t-d_{1}\right)\right]-d_{12} \int_{t-d_{2}}^{t-d_{1}} \dot{\tilde{x}}^{T}(\alpha) \widetilde{E}^{T} \Phi^{T} R_{2} \Phi \widetilde{E} \dot{\tilde{x}}(\alpha) d \alpha \\
= & -d_{12} \int_{t-d(t)}^{t-d_{1}} \dot{\tilde{x}}^{T}(\alpha) \widetilde{E}^{T} \Phi^{T} R_{2} \Phi \widetilde{E} \dot{\tilde{x}}(\alpha) d \alpha-d_{12} \int_{t-d_{2}}^{t-d(t)} \dot{\tilde{x}}^{T}(\alpha) \widetilde{E}^{T} \Phi^{T} R_{2} \Phi \widetilde{E} \dot{\tilde{x}}(\alpha) d \alpha \\
\leq & -\frac{d_{12}}{d(t)-d_{1}}\left[\int_{t-d(t)}^{t-d_{1}} \Phi \widetilde{E} \dot{\tilde{x}}(\alpha) d \alpha\right]^{T} R_{2}\left[\int_{t-d(t)}^{t-d_{1}} \Phi \widetilde{E} \dot{\tilde{x}}(\alpha) d \alpha\right]-\frac{d_{12}}{d_{2}-d(t)} \\
& \cdot\left[\int_{t-d_{2}}^{t-d(t)} \Phi \widetilde{E} \dot{\tilde{x}}(\alpha) d \alpha\right]^{T} R_{2}\left[\int_{t-d_{2}}^{t-d(t)} \Phi \widetilde{E} \dot{\tilde{x}}(\alpha) d \alpha\right]
\end{aligned}
$$

Next, let us estimate the upper bound of the two terms on the right side of the above inequality.

When $0<d_{1}<d(t)<d_{2}$, there is

$$
d(t)-d_{1}<d_{2}-d_{1} \Longrightarrow\left\{\begin{array}{l}
\frac{d_{2}-d_{1}}{d(t)-d_{1}}>1 \Longrightarrow-\frac{d_{2}-d_{1}}{d(t)-d_{1}}<-1, \\
\frac{d_{2}-d_{1}}{d_{2}-d(t)}>1 \Longrightarrow-\frac{d_{2}-d_{1}}{d_{2}-d(t)}<-1 .
\end{array}\right.
$$

Therefore, the following can be obtained:

$$
\begin{aligned}
& \delta V_{4}(\widetilde{x}(t), i, t) \\
& \left.\left.<\dot{\tilde{x}}^{T}(t) \widetilde{E}^{T} \Phi^{T}\left(d_{1}^{2} R_{1}+d_{12}^{2} R_{2}\right) \Phi \widetilde{E} \dot{\tilde{x}}(t)-\left(\int_{t-d_{1}}^{t} \Phi \widetilde{E} \dot{\tilde{x}}(\alpha) d \alpha\right)\right)^{T} R_{1}\left(\int_{t-d_{1}}^{t} \Phi \widetilde{E} \dot{\tilde{x}}(\alpha) d \alpha\right)\right) \\
& -\left(\int_{t-d(t)}^{t-d_{1}} \Phi \widetilde{E} \dot{\bar{x}}(\alpha) d \alpha\right)^{T} R_{2}\left(\int_{t-d(t)}^{t-d_{1}} \Phi \widetilde{E} \dot{\bar{x}}(\alpha) d \alpha\right)-\left(\int_{t-d_{2}}^{t-d(t)} \Phi \widetilde{E} \dot{\tilde{x}}(\alpha) d \alpha\right)^{T} R_{2}\left(\int_{t-d_{2}}^{t-d(t)} \Phi \widetilde{E} \dot{\tilde{x}}(\alpha) d \alpha\right) \\
& =\dot{\tilde{x}}^{T}(t) \widetilde{E}^{T} \Phi^{T}\left(d_{1}^{2} R_{1}+d_{12}^{2} R_{2}\right) \Phi \widetilde{E} \dot{\bar{x}}(t)-\left[\widetilde{x}(t)-\widetilde{x}\left(t-d_{1}\right)\right]^{T} \widetilde{E}^{T} \Phi^{T} R_{1} \Phi \widetilde{E}\left[\widetilde{x}(t)-\widetilde{x}\left(t-d_{1}\right)\right] \\
& -\left[\widetilde{x}\left(t-d_{1}\right)-\widetilde{x}(t-d(t))\right]^{T} \widetilde{E}^{T} \Phi^{T} R_{2} \Phi \widetilde{E}\left[\widetilde{x}\left(t-d_{1}\right)-\widetilde{x}(t-d(t))\right] \\
& -\left[\widetilde{x}(t-d(t))-\tilde{x}\left(t-d_{2}\right)\right]^{T} \widetilde{E}^{T} \Phi^{T} R_{2} \Phi \widetilde{E}\left[\widetilde{x}(t-d(t))-\widetilde{x}\left(t-d_{2}\right)\right] \\
& =\left[\widetilde{A}_{i} \widetilde{x}(t)+\widetilde{A}_{d i} \Phi \widetilde{x}(t-d(t))\right]^{T} \Phi^{T}\left(d_{1}^{2} R_{1}+d_{12}^{2} R_{2}\right) \Phi\left[\widetilde{A}_{i} \widetilde{x}(t)+\widetilde{A}_{d i} \Phi \widetilde{x}(t-d(t))\right] \\
& -\left[\tilde{x}(t)-\tilde{x}\left(t-d_{1}\right)\right]^{T} \widetilde{E}^{T} \Phi^{T} R_{1} \Phi \widetilde{E}\left[\tilde{x}(t)-\tilde{x}\left(t-d_{1}\right)\right] \\
& -\left[\widetilde{x}\left(t-d_{1}\right)-\tilde{x}(t-d(t))\right]^{T} \widetilde{E}^{T} \Phi^{T} R_{2} \Phi \widetilde{E}\left[\tilde{x}\left(t-d_{1}\right)-\tilde{x}(t-d(t))\right] \\
& -\left[\tilde{x}(t-d(t))-\tilde{x}\left(t-d_{2}\right)\right]^{T} \widetilde{E}^{T} \Phi^{T} R_{2} \Phi \widetilde{E}\left[\tilde{x}(t-d(t))-\tilde{x}\left(t-d_{2}\right)\right] .
\end{aligned}
$$


Noting that $\widetilde{E}^{T} \widetilde{S}=0$ and $\widetilde{E} \widetilde{N}=0$, we have

$$
\begin{aligned}
& \nabla V(\tilde{x}(t), i, t) \\
& =\dot{\tilde{x}}^{T}(t) \widetilde{E}^{T} \widetilde{S} \widetilde{N}^{T} \widetilde{x}(t)+\widetilde{x}^{T}(t) \tilde{N} \widetilde{S}^{T} \widetilde{E} \dot{\tilde{x}}(t) \\
& =\left[\widetilde{A}_{i} \widetilde{x}(t)+\widetilde{A}_{d i} \Phi \widetilde{x}(t-d(t))\right]^{T} \widetilde{S} \widetilde{N}^{T} \widetilde{x}(t)+\widetilde{x}^{T}(t) \widetilde{N} \widetilde{S}^{T}\left[\widetilde{A}_{i} \widetilde{x}(t)+\widetilde{A}_{d i} \Phi \widetilde{x}(t-d(t))\right]=0 .
\end{aligned}
$$

Add the weak infinitesimal operators to get the following equation:

$$
\begin{aligned}
& \delta V(\tilde{x}(t), i, t) \\
& =\delta V_{1}(\tilde{x}(t), i, t)+\delta V_{2}(\tilde{x}(t), i, t)+\delta V_{3}(\tilde{x}(t), i, t)+\delta V_{4}(\tilde{x}(t), i, t)+\nabla V(\tilde{x}(t), i, t) \\
& \leq \widetilde{x}^{T}(t)\left(\sum_{j=1}^{N} \lambda_{i j} \widetilde{E}^{T} P_{j} \widetilde{E}\right) \widetilde{x}(t)+\widetilde{x}^{T}(t) \widetilde{E}^{T} P_{i}\left[\widetilde{A}_{i} \tilde{x}(t)+\widetilde{A}_{d i} \Phi \tilde{x}(t-d(t))\right]+\left[\widetilde{A}_{i} \widetilde{x}(t)+\widetilde{A}_{d i} \Phi \tilde{x}(t-d(t))\right]^{T} P_{i}^{T} \widetilde{E} \tilde{x}(t) \\
& +\tilde{x}^{T}(t) \Phi^{T}\left(Q_{1 i}+Q_{2 i}+Q_{3 i}\right) \Phi \tilde{x}(t)-(1-u) \widetilde{x}^{T}(t-d(t)) \Phi^{T} Q_{1 i} \widetilde{E} \tilde{x}(t-d(t))-\tilde{x}^{T}\left(t-d_{1}\right) \Phi^{T} Q_{2 i} \widetilde{E} \tilde{x}\left(t-d_{1}\right) \\
& -\tilde{x}^{T}\left(t-d_{2}\right) \Phi^{T} Q_{3 i} \widetilde{E} \tilde{x}\left(t-d_{2}\right)+\int_{t-d(t)}^{t} \tilde{x}^{T}(\alpha) \Phi^{T}\left(\sum_{j=1}^{N} \lambda_{i j} Q_{1 j}\right) \Phi \tilde{x}(\alpha) d \alpha+\int_{t-d_{1}}^{t} \tilde{x}^{T}(\alpha) \Phi^{T}\left(\sum_{j=1}^{N} \lambda_{i j} Q_{2 j}\right) \Phi \tilde{x}(\alpha) d \alpha \\
& +\int_{t-d_{2}}^{t-d(t)} \tilde{x}^{T}(\alpha) \Phi^{T}\left(\sum_{j=1}^{N} \lambda_{i j} Q_{3 j}\right) \Phi \tilde{x}(\alpha) d \alpha+\int_{t-d(t)}^{t} \tilde{x}^{T}(\alpha) \Phi^{T}\left(\sum_{j=1}^{N} \lambda_{i j} Q_{3 j}\right) \Phi \tilde{x}(\alpha) d \alpha+\tilde{x}^{T}(t) \Phi^{T}\left[d_{1} Z_{1}+d_{2} Z_{2}\right] \Phi \tilde{x}(t) \\
& -\int_{t-d_{1}}^{t} \tilde{x}^{T}(\alpha) \Phi^{T} Z_{1} \Phi \tilde{x}(\alpha) d \alpha-\int_{t-d_{2}}^{t} \tilde{x}^{T}(\alpha) \Phi^{T} Z_{2} \Phi \tilde{x}(\alpha) d \alpha+\left[\tilde{A}_{i} \tilde{x}(t)+\widetilde{A}_{d i} \Phi \tilde{x}(t-d(t))\right]^{T} \Phi^{T}\left(d_{1}^{2} R_{1}+d_{12}^{2} R_{2}\right) \Phi \\
& \cdot\left[\widetilde{A}_{i} \widetilde{x}(t)+\widetilde{A}_{d i} \Phi \widetilde{x}(t-d(t))\right]-\left[\widetilde{x}(t)-\widetilde{x}\left(t-d_{1}\right)\right]^{T} \widetilde{E}^{T} \Phi^{T} R_{1} \Phi \widetilde{E}\left[\widetilde{x}(t)-\widetilde{x}\left(t-d_{1}\right)\right] \\
& -\left[\tilde{x}\left(t-d_{1}\right)-\tilde{x}(t-d(t))\right]^{T} \widetilde{E}^{T} \Phi^{T} R_{2} \Phi \widetilde{E}\left[\tilde{x}\left(t-d_{1}\right)-\tilde{x}(t-d(t))\right] \\
& \left.-\left[\tilde{x}(t-d(t))-\tilde{x}(t)-d_{2}\right)\right]^{T} \widetilde{E}^{T} \Phi^{T} R_{2} \Phi \widetilde{E}\left[\widetilde{x}(t-d(t))-\tilde{x}\left(t-d_{2}\right)\right]+\dot{\tilde{x}}^{T}(t) \widetilde{E}^{T} \widetilde{S}^{N} \tilde{N}^{T} \tilde{x}(t)+\tilde{x}^{T}(t) \tilde{N} \widetilde{S}^{T} \widetilde{E} \dot{\tilde{x}}(t) \\
& =\xi_{1}^{T}(t) \Omega_{1 i} \xi_{1}(t),
\end{aligned}
$$

where

$$
\begin{aligned}
& \xi_{1}(t)=\left[\begin{array}{llll}
\widetilde{x}^{T}(t) & \tilde{x}^{T}(k-d(t)) & \tilde{x}^{T}\left(t-d_{1}\right) & \tilde{x}^{T}\left(t-d_{2}\right)
\end{array}\right]^{T}, \\
& \Omega_{1 i}=\left[\begin{array}{cccc}
\Omega_{11}^{\prime} & \Omega_{12}^{\prime} & \widetilde{E}^{T} \Phi^{T} R_{1} \Phi \widetilde{E} & 0 \\
* & \Omega_{22}^{\prime} & \widetilde{E}^{T} \Phi^{T} R_{2} \Phi \widetilde{E} & \widetilde{E}^{T} \Phi^{T} R_{2} \Phi \widetilde{E} \\
* & * & \Omega_{33}^{\prime} & 0 \\
* & * & * & \Omega_{44}^{\prime}
\end{array}\right], \\
& \Omega_{11}^{\prime}=\sum_{j=1}^{N} \lambda_{i j} \widetilde{E}^{T} P_{j} \widetilde{E}+\operatorname{sym}\left(\widetilde{E}^{T} P_{i} \widetilde{A}_{i}\right)+\operatorname{sym}\left(\widetilde{A}_{i}^{T} \widetilde{S}_{N_{i}^{T}}^{T}\right)+\Phi^{T}\left(Q_{1 i}+Q_{2 i}+Q_{3 i}+d_{1} Z_{1}+d_{2} Z_{2}\right) \Phi \\
& -\widetilde{E}^{T} \Phi^{T} R_{1} \Phi \widetilde{E}+\widetilde{A}_{i}^{T} \Phi^{T}\left(d_{1}^{2} R_{1}+d_{12}^{2} R_{2}\right) \Phi \widetilde{A}_{i} \\
& \Omega_{12}^{\prime}=\widetilde{E}^{T} P_{i} \widetilde{A}_{d i} \Phi+\tilde{N}_{i} \widetilde{S}^{T} \widetilde{A}_{d i} \Phi+\widetilde{A}_{i}^{T} \Phi^{T}\left(d_{1}^{2} R_{1}+d_{12}^{2} R_{2}\right) \Phi \widetilde{A}_{d i} \Phi, \\
& \Omega_{22}^{\prime}=-(1-u) \Phi^{T} Q_{1 i} \Phi-2 \widetilde{E}^{T} \Phi^{T} R_{2} \Phi \widetilde{E}+\Phi^{T} \widetilde{A}_{d i}^{T} \Phi^{T}\left(d_{1}^{2} R_{1}+d_{12}^{2} R_{2}\right) \Phi \widetilde{A}_{d i} \Phi \text {, } \\
& \Omega_{33}^{\prime}=-\Phi^{T} Q_{2 i} \Phi-\widetilde{E}^{T} \Phi^{T}\left(R_{1}+R_{2}\right) \Phi \widetilde{E} \\
& \Omega_{44}^{\prime}=-\Phi^{T} Q_{3 i} \Phi-\widetilde{E}^{T} \Phi^{T} R_{2} \Phi \widetilde{E} \text {. }
\end{aligned}
$$


If $\delta V(\tilde{x}(t), i, t)=\xi_{1}^{T}(t) \Omega_{1 i} \xi_{1}(t)<0$ is true, system (9) is stochastically stable. Next, we derive the conditions for $\delta V(\tilde{x}(t), i, t)<0$ to be true. Let the minimum eigenvalue of matrix $-\Psi_{i}$ be $\lambda_{\min }\left(-\Psi_{i}\right)$. According to equation (28), the following equation can be obtained:

$$
\delta V(\widetilde{x}(t), i, t)<-\lambda_{\min }\left(-\Psi_{i}\right)^{T} \widetilde{x}^{T}(t) \tilde{x}(t) .
$$

According to Dynkin formula, we can get

$$
\begin{aligned}
E[V(\widetilde{x}(t)), r(t)]-V\left(\widetilde{x}_{0}, r_{0}\right) & =E\left[\int_{0}^{t} \delta V(\widetilde{x}(s), r(s), s) d s\right] \\
& \leq-\lambda_{\min }\left(-\Psi_{i}\right) E\left[\int_{0}^{t} \tilde{x}^{T}(s) \widetilde{x}(s) d s \mid \tilde{x}_{0}, r_{0}\right]
\end{aligned}
$$

from $V(x(t), r(t), t) \geq 0$; when $\delta V(\tilde{x}(t), r(t), t)<0$, the following formula is true:

$$
E\left[\int_{0}^{t} \tilde{x}^{T}(s) \widetilde{x}(s) d s \mid \tilde{x}_{0}, r_{0}\right] \leq \frac{V\left(\widetilde{x}_{0}, r_{0}\right)}{\lambda_{\min }\left(-\Psi_{i}\right)}=M\left(\widetilde{x}_{0}, r_{0}\right) .
$$

When the upper limit of the integration tends to infinity, it can be deduced:

$$
E\left[\int_{0}^{\infty} \tilde{x}^{T}(s) \tilde{x}(s) d s \mid \tilde{x}_{0}, r_{0}\right] \leq M\left(\widetilde{x}_{0}, r_{0}\right) .
$$

As can be seen from the definition of 1.1 (3), system (9) is stochastically stable. System (9) is regular, impulse-free, and stochastically stable; thus, it is stochastically admissible.

When $\Psi(t) \neq 0$, set

$$
\xi_{2}(t)=\left[\begin{array}{lllll}
\tilde{x}^{T}(t) & \tilde{x}^{T}(k-d(t)) & \tilde{x}^{T}\left(t-d_{1}\right) & \tilde{x}^{T}\left(t-d_{2}\right) & \Psi^{T}(t)
\end{array}\right]^{T} .
$$

According to (2) in Definition 2, the following formula can be obtained:

$$
\begin{aligned}
& J(T) \\
& \leq E\left\{\int_{0}^{T}\left[e^{T}(t) e(t)-\gamma^{2} \Psi^{T}(t) \Psi(t)+\delta V(\tilde{x}(t), r(t), t)\right] d t\right\}-E\left\{\int_{0}^{T}[\delta V(\tilde{x}(t), r(t), t)] d t\right\} \\
& \leq E\left\{\int_{0}^{T}\left[e^{T}(t) e(t)-\gamma^{2} \Psi^{T}(t) \Psi(t)+\delta V(\widetilde{x}(t), r(t), t)\right] d t\right\} \\
& \leq E\left\{\int_{0}^{T} \xi_{2}^{T}(t) \Omega_{2 i} \xi_{2}(t) d t\right\} \\
& \Omega_{2 i}=\left[\begin{array}{ccccc}
\widetilde{\Omega}_{11} & \widetilde{\Omega}_{12} & \widetilde{E}^{T} \Phi^{T} R_{1} \Phi \widetilde{E} & 0 & \Omega_{15} \\
* & \widetilde{\Omega}_{22} & \widetilde{E}^{T} \Phi^{T} R_{2} \Phi \widetilde{E} & \widetilde{E}^{T} \Phi^{T} R_{2} \Phi \widetilde{E} & \Omega_{25} \\
* & * & \Omega_{33}^{\prime} & 0 & 0 \\
* & * & * & \Omega_{44}^{\prime} & 0 \\
* & * & * & * & \Omega_{55}
\end{array}\right], \\
& \widetilde{\Omega}_{11}=\Omega_{11}^{\prime}+\widetilde{C}_{T}^{i} \widetilde{C}_{i} \text {, } \\
& \widetilde{\Omega}_{12}=\Omega_{12}^{\prime}+\widetilde{C}_{T}^{i} \widetilde{C}_{d i} \Phi, \\
& \widetilde{\Omega}_{22}=\Omega_{22}^{\prime}+\Phi^{T} \widetilde{C}_{T}^{d i} \widetilde{C}_{d i} \Phi, \\
& \Omega_{15}=\widetilde{E}^{T} P_{i}^{T} \widetilde{B}_{i}+\widetilde{A}_{i}^{T} \Phi^{T}\left(d_{1}^{2} R_{1}+d_{12}^{2} R_{2}\right) \Phi \widetilde{B}_{i}+\widetilde{C}_{i}^{T} \widetilde{D}_{i}+\widetilde{N}_{i} \widetilde{S}^{T} \widetilde{B}_{i}, \\
& \Omega_{25}=\Phi^{T} \widetilde{A}_{d i}^{T} \Phi^{T}\left(d_{1}^{2} R_{1}+d_{12}^{2} R_{2}\right) \Phi \widetilde{B}_{i}+\Phi^{T} \widetilde{C}_{d i}^{T} \widetilde{D}_{i} \text {, } \\
& \Omega_{55}=\widetilde{B}_{i}^{T} \Phi^{T}\left(d_{1}^{2} R_{1}+d_{12}^{2} R_{2}\right) \Phi \widetilde{B}_{i}+\widetilde{D}_{T}^{i} \widetilde{D}_{i}-\gamma^{2} I .
\end{aligned}
$$

Applying the Schur theorem to formula (35), formula (18) can be obtained. Theorem 1 is proved.

Theorem 2. Considering singular model error augmented system (9) with polytopic uncertain transition rates and time-varying delays is stochastic admissible with an $\mathrm{H}_{\mathrm{o}}$ performance index $\gamma$. If there exist positivedefinite symmetric matrices $P_{i} \in R^{\left(n_{x}+n_{f}+n_{w}\right) \times\left(n_{x}+n_{f}+n_{w}\right)}$, $\left\{Z_{1}, Z_{2}, R_{1}, R_{2}, Q_{1 i}, Q_{2 i}, Q_{3 i}\right\} \in R^{n_{x} \times n_{x}}$, any appropriate dimensions matrices $\widetilde{S}, \widetilde{N}_{i} \in R^{\left(n_{x}+n_{f}+n_{w}\right) \times\left(n_{x}-r\right)}$, and satisfying $\widetilde{E}^{T} \widetilde{S}=0$, such that the following LMIs hold for any $\forall i, j \in I$ : 


$$
\begin{aligned}
& \delta_{1 k}^{(i)}=\sum_{j \in S_{k}^{(i)}} \lambda_{i j} Q_{1 j}+\sum_{j \in S_{u c}^{(i)}} \tilde{\lambda}_{i j}^{(l)} Q_{1 j}, \\
& \delta_{2 k}^{(i)}=\sum_{j \in S_{k}^{(i)}} \lambda_{i j} Q_{2 j}+\sum_{j \in S_{u c}^{(i)}} \tilde{\lambda}_{i j}^{(l)} Q_{2 j}, \\
& \delta_{3 k}^{(i)}=\sum_{j \in S_{k}^{(i)}} \lambda_{i j} Q_{3 j}+\sum_{j \in S_{u c}^{(i)}} \tilde{\lambda}_{i j}^{(l)} Q_{3 j} .
\end{aligned}
$$

If $i \in S_{k}^{(i)} \cup S_{u c}^{(i)}, j \in S_{u k}^{(i)}$,

$$
\left\{\begin{array}{l}
\delta_{1 k}^{(i)}+\delta_{3 k}^{(i)}-\left(\lambda_{k}^{i}+\lambda_{u c}^{(i l)}\right)\left(Q_{1 j}+Q_{3 j}\right)<Z_{2}, \\
\delta_{2 k}^{(i)}-\left(\lambda_{k}^{i}+\lambda_{u c}^{(i l)}\right) Q_{2 j}<Z_{1}, \\
\delta_{3 k}^{(i)}-\left(\lambda_{k}^{i}+\lambda_{u c}^{(i l)}\right) Q_{3 j}<Z_{2} .
\end{array}\right.
$$

If $i \in S_{u k}^{(i)}, j \in S_{u k}^{(i)}$,

$$
\begin{aligned}
& \widehat{\Omega}_{11}=\operatorname{sym}\left(\widetilde{E}^{T} P_{i} \widetilde{A}_{i}\right)+\operatorname{sym}\left(\widetilde{A}_{i}^{T} \widetilde{S} \widetilde{N}_{i}^{T}\right)+\Phi^{T}\left(Q_{1 i}+Q_{2 i}+Q_{3 i}+d_{1} Z_{1}+d_{2} Z_{2}\right) \Phi-\widetilde{E}^{T} \Phi^{T} R_{1} \Phi \widetilde{E}, \\
& \widehat{\Omega}_{11}=\widehat{\Omega}_{11}+\widetilde{E}^{T}\left[\Xi_{k}^{(i)}+\Xi_{u c}^{(i l)}-\left(\lambda_{k}^{(i)}+\lambda_{u c}^{(i)}\right) P_{j}\right] \widetilde{E}, \text { if } i \in S_{k}^{(i)} \cup S_{u c}^{(i)}, j \in S_{u k}^{(i)}, \\
& \widehat{\Omega}_{11}=\widehat{\Omega}_{11}+\widetilde{E}^{T}\left[\Xi_{k}^{(i)}+\Xi_{u c}^{(i l)}+\lambda_{b}^{i} P_{i}-\left(\lambda_{b}^{i}+\lambda_{k}^{(i)}+\lambda_{u c}^{(i l)}\right) P_{j}\right] \widetilde{E}, \text { if } i \in S_{u k}^{(i)}, j \in S_{u k}^{(i)}, \\
& \Xi_{k}^{(i)}=\sum_{j \in S_{k}^{(i)}} \lambda_{i j} P_{j}, \Xi_{u c}^{(i)}=\sum_{j \in S_{u c}^{(i)}} \widetilde{\lambda}_{i j}^{(l)} P_{j}, \lambda_{k}^{(i)}=\sum_{j \in S_{k}^{(i)}} \lambda_{i j}, \lambda_{u c}^{(i)}=\sum_{j \in S_{u c}^{(i)}} \widetilde{\lambda}_{i j}^{(l)} .
\end{aligned}
$$

Proof. Theorem 1 has given the sufficient condition that system (9) is stochastically admissible and has an $H_{\infty}$ performance indexes $\gamma$ when the TRs are completely known. Next, we discuss the case that the state TRM contains unknown elements.

Case 1. If $i \in S_{k}^{(i)} \cup S_{u c}^{(i)}, j \in S_{u k}^{(i)}$.
When $i \in S_{k}^{(i)} \cup S_{u c}^{(i)}$, it is indicated that diagonal elements $\lambda_{i i}$ are known or uncertain, then $\lambda_{k}^{(i)}+\lambda_{u c}^{(i l)} \leq 0$. Since $\lambda_{k}^{(i)}+\lambda_{u c}^{(i l)}=0$, all the elements in the ith row of the vertex $\Lambda_{l}, l=1,2, \cdots M$ are known, we only need to consider the case $\lambda_{k}^{(i)}+\lambda_{u c}^{(i l)}<0$ and rewrite the term $\sum_{j=1}^{N} \lambda_{i j} P_{j}$ in formula (18) as

$$
\begin{aligned}
\sum_{j=1}^{N} & \lambda_{i j} P_{j}=\sum_{j \in S_{k}^{(i)}} \lambda_{i j} P_{j}+\sum_{j \in S_{u k}^{(i)}} \hat{\lambda}_{i j} P_{j}+\sum_{j \in S_{u c}^{(i)}}\left(\sum_{l=1}^{M} \alpha_{l} \tilde{\lambda}_{i j}^{(l)}\right) P_{j} \\
& =\sum_{j \in S_{k}^{(i)}} \lambda_{i j} P_{j}+\left(-\lambda_{k}^{(i)}-\lambda_{u c}^{(i l)}\right) \sum_{j \in S_{u k}^{(i)}} \frac{\lambda_{i j}^{(i)}-\lambda_{u c}^{(i l)}}{P_{j}}+\sum_{l=1}^{M} \alpha_{l} \sum_{j \in S_{u c}^{(i)}} \tilde{\lambda}_{i j}^{(l)} P_{j} \\
& =\Xi_{k}^{(i)}+\left(-\lambda_{k}^{(i)}-\lambda_{u c}^{(i l)}\right) \sum_{j \in S_{u k}^{(i)}} \frac{\widehat{\lambda}_{i j}^{(i)}}{-\lambda_{k}^{(i)}-\lambda_{u c}^{(i l)}} P_{j}+\sum_{l=1}^{M} \alpha_{l} \Xi_{u c}^{(i l)} \\
& =\Xi_{k}^{(i)}+\left(-\lambda_{k}^{(i)}-\lambda_{u c}^{(i l)}\right) \sum_{j \in S_{u k}^{(i)}} \frac{\hat{\lambda}_{i j}}{-\lambda_{k}^{(i)}-\lambda_{u c}^{(i l)}} P_{j}+\sum_{l=1}^{M} \alpha_{l} \Xi_{u c}^{(i l)},
\end{aligned}
$$


where $\hat{\lambda}_{i j}\left(j \in S_{u k}^{(i)}\right)$ and $\tilde{\lambda}_{i j}\left(j \in S_{u c}^{(i)}\right)$ represent unknown and polytopic uncertain elements, respectively, $\Xi_{k}^{(i)}=\sum_{j \in S_{k}^{(i)}} \lambda_{i j} P_{j}$, and $\Xi_{u c}^{(i l)}=\sum_{j \in S_{u c}^{(i)}} \tilde{\lambda}_{i j}^{(l)} P_{j}$. Since $0 \leq \alpha_{l} \leq 1, \quad \sum_{l=1}^{M} \alpha_{l}=1$, and $0 \leq \widehat{\lambda}_{i j} /-\lambda_{k}^{(i)}-\lambda_{u c}^{(i l)} \leq 1, \sum_{j \in S_{u k}^{(i)}} \widehat{\lambda}_{i j} /-\lambda_{k}^{(i)}-\lambda_{u c}^{(i l)}=1$, the right side (RS) of formula (40) can be written as

$$
R S(50)=\sum_{l=1}^{M} \alpha_{l} \sum_{j \in S_{u k}^{(i)}} \frac{\hat{\lambda}_{i j}}{-\lambda_{k}^{(i)}-\lambda_{u c}^{(i l)}}\left(\Xi_{k}^{(i)}+\Xi_{u c}^{(i l)}-\left(-\lambda_{k}^{(i)}-\lambda_{u c}^{(i l)}\right) P_{j}\right) .
$$

Similarly, when $0 \leq \alpha_{l} \leq 1$ and $0 \leq \widehat{\lambda}_{i j} \leq-\left(\lambda_{k}^{(i)}+\lambda_{u c}^{(i l)}\right)$, the left side (LS) of formula (18) can be transformed into the following form:

$$
\operatorname{LHS}(18)=\sum_{l=1}^{M} \alpha_{l} \sum_{j \in S_{u k}^{(i)}} \frac{\widehat{\lambda}_{i j}}{-\lambda_{k}^{(i)}-\lambda_{u c}^{(i l)}} \widehat{\Omega}_{i}, \quad j \in S_{u k}^{(i)}, l=1,2, \cdots M,
$$

where $\widehat{\Omega}_{i}$ has been defined in formula (39), which means that when not all elements are known in the TRM, the inequality set (17) is directly converted into the inequality set (37).

Case 2. If $i \in S_{u k}^{(i)}, j \in S_{u k}^{(i)}$.

When $0 \leq \alpha_{l} \leq 1, \hat{\lambda}_{i i}=-\left(\lambda_{k}^{(i)}+\lambda_{u c}^{(i l)}\right)$, the elements in the $i$ th row are all known, so $0 \leq \alpha_{l} \leq 1, \hat{\lambda}_{i i}<-\left(\lambda_{k}^{(i)}+\lambda_{u c}^{(i l)}\right)$ is true if at least one unknown element in the ith row exists besides the diagonal. Under this condition, the $0 \leq \alpha_{l} \leq 1, \sum_{j=1}^{N} \lambda_{i j} P_{j}$ term in formula (18) can be rewritten as

$$
\begin{aligned}
\sum_{j=1}^{N} & \lambda_{i j} P_{j} \\
= & \sum_{j \in S_{k}^{(i)}} \lambda_{i j} P_{j}+\hat{\lambda}_{i i} P_{i}+\sum_{j \in S_{u k}^{(i)}, j \neq i} \hat{\lambda}_{i j} P_{j}+\sum_{j \in S_{u c}^{(i)}}\left(\sum_{l=1}^{M} \alpha_{l} \widetilde{\lambda}_{i j}^{(l)}\right) P_{j} \\
= & \Xi_{k}^{(i)}+\hat{\lambda}_{i i} P+\left(-\hat{\lambda}_{i i}-\lambda_{k}^{(i)}-\lambda_{u c}^{(i l)}\right) \sum_{j \in S_{u k}^{(i)}, j \neq i} \\
& \cdot \frac{\hat{\lambda}_{i j}}{-\hat{\lambda}_{i i}-\lambda_{k}^{(i)}-\lambda_{u c}^{(i l)}} P_{j}+\sum_{l=1}^{M} \alpha_{l} \Xi_{u c}^{(i l)} .
\end{aligned}
$$

Since $\quad 0 \leq \alpha_{l} \leq 1, \quad \sum_{l=1}^{M} \alpha_{l}=1, \quad$ and $0 \leq \widehat{\lambda}_{i j} /-\hat{\lambda}_{i i}-\lambda_{k}^{(i)}-\lambda_{u c}^{(i l)} \leq 1, \quad \sum_{j \in S_{u k}^{(i)}, j \neq i} \hat{\lambda}_{i j} /-\hat{\lambda}_{i i}-\lambda_{k}^{(i)}-\lambda_{u c}^{(i l)}$ $=1$; the following formula can be deduced:

$$
\begin{aligned}
\sum_{j=1}^{N} \lambda_{i j} P_{j}= & \sum_{l=1}^{M} \alpha_{l} \sum_{j \in S_{u k}^{(i)}, j \neq i} \frac{\hat{\lambda}_{i j}}{-\hat{\lambda}_{i i}-\lambda_{k}^{(i)}-\lambda_{u c}^{(i l)}} \\
& \cdot\left[\Xi_{k}^{(i)}+\hat{\lambda}_{i i} P_{i}+\Xi_{u c}^{(i l)}-\left(\hat{\lambda}_{i i}+\lambda_{k}^{(i)}+\lambda_{u c}^{(i)}\right) P_{j}\right] .
\end{aligned}
$$

By rewriting the left side (LS) of inequality (18) according to equation (44), we can get

$$
L S(18)=\sum_{l=1}^{M} \alpha_{l} \sum_{j \in S_{u k}^{(i)}, j \neq i} \frac{\widehat{\lambda}_{i j}}{-\widehat{\lambda}_{i i}-\lambda_{k}^{(i)}-\lambda_{u c}^{(i)}} \widehat{\Omega}_{i}, \quad i \in S_{u k}^{(i)}, j \in S_{u k}^{(i)}
$$

$$
\begin{aligned}
\widehat{\Omega}_{11}= & \widehat{\Omega}_{11}+\widetilde{E}^{T}\left[\Xi_{k}^{(i)}+\Xi_{u c}^{(i l)}+\widehat{\lambda}_{i i} P_{i}-\left(\widehat{\lambda}_{i i}+\lambda_{k}^{(i)}\right.\right. \\
& \left.\left.+\lambda_{u c}^{(i l)}\right) P_{j}\right] \widetilde{E}
\end{aligned}
$$

where $\widehat{\Omega}_{i}$ has been defined in formula (39) and contains the term $\widetilde{\Omega}_{11}$. In order to achieve the solvability of the stability analysis, the lower bound of the unknown element $\widehat{\lambda}_{i i}$ is defined as $\lambda_{b}^{(i)}$, and $\lambda_{b}^{(i)} \leq \widehat{\lambda}_{i i}<-\lambda_{k}^{(i)}-\lambda_{u c}^{(i l)}$ can be obtained. It indicates that, for any small $\varepsilon<0, \widehat{\lambda}_{i i}$ can take value in the interval $\left[\lambda_{b}^{(i)},-\lambda_{k}^{(i)}-\lambda_{u c}^{(i l)}+\varepsilon\right]$, then $\hat{\lambda}_{i i}$ can be written as the form of the following convex combination $\widehat{\lambda}_{i i}=-\beta \lambda_{k}^{(i)}-\beta \lambda_{u c}^{(i l)}+\beta \varepsilon+(1-\beta) \lambda_{b}^{(i)}$, where $0 \leq \beta \leq 1$, and it can be seen that $\widehat{\lambda}_{i i}$ depends linearly on $\beta$. Therefore, if only $\beta$ takes the boundary value of 0 or 1 that the linear solvable condition of inequality (45) can be satisfied. At this time, equation (46) can be rewritten as

$$
\begin{array}{r}
\widehat{\Omega}_{11}=\widehat{\Omega}_{11}+\widetilde{E}^{T}\left[\Xi_{k}^{(i)}+\Xi_{u c}^{(i)}+\lambda_{b}^{i} P_{i}-\left(\lambda_{b}^{i}+\lambda_{k}^{(i)}+\lambda_{u c}^{(i l)}\right) P_{j}\right] \widetilde{E}, \\
\beta=0, \quad j \neq i,
\end{array}
$$

$$
\begin{array}{r}
\widehat{\Omega}_{11}=\widehat{\Omega}_{11}+\widetilde{E}^{T}\left[\Xi_{k}^{(i)}+\Xi_{u c}^{(i l)}-\left(\lambda_{k}^{(i)}+\lambda_{u c}^{(i l)}\right) P_{j}+\varepsilon\left(P_{i}-P_{j}\right)\right] \widetilde{E}, \\
\beta=1, \quad j \neq i .
\end{array}
$$

When $\varepsilon$ is infinitely small and $j \neq i, j \in S_{u k}^{(i)}$, formula (48) can be converted to

$$
\widehat{\Omega}_{11}=\widehat{\Omega}_{11}+\widetilde{E}^{T}\left[\Xi_{k}^{(i)}+\Xi_{u c}^{(i l)}-\left(\lambda_{k}^{(i)}+\lambda_{u c}^{(i l)}\right) P_{j}\right] \widetilde{E}, \quad j \neq i .
$$

When $j=i, j \in S_{u k}^{(i)}$,equation (47) can be written as (49), so when $i \in S_{u k}^{(i)}$ and $j \in S_{u k}^{(i)}$, the term $\widehat{\Omega}_{11}$ can be expressed by (47). The inequality group (17) can be directly converted into the inequality group (38). Therefore, when the elements in the state TRM are not completely known, as long as the inequality set (37)-(39) exists, system (9) is still stochastically admissible and has an $H_{\infty}$ performance $\gamma$. The prove is completed.

Remark 4. In this section, stochastically admissible analysis criteria for a family of polytopic uncertain singular continuous-time Markovian jump error augmented systems with time-varying delays are given. Because the coupling terms in the system matrices will affect the performance of the FDFs, we will use a special method to design the fullorder and reduced-order FDFs of polytopic uncertain CTSMJSs with time-varying delays. It can improve the effectiveness and practicability of the FDFs.

\section{Design of Full-Order and Reduced-Order FDFS for Polytopic Uncertain CTSMJSS with Time-Varying Delays}

Theorem 3. On the premise that the error augmented system (9) is stochastically admissible and has an $H_{\infty}$ performance index $\gamma$, for a given scalar $\gamma>0$, if exist positive definite symmetric 
matrices $P_{i}:=\left[\begin{array}{ccc}P_{i(1)} & K P_{i(2)} & P_{i(4)} \\ * & P_{i(2)} & 0 \\ * & * & P_{i(5)}\end{array}\right] \in R^{\left(n_{x}+n_{f}+n_{w}\right) \times\left(n_{x}+n_{f}+n_{w}\right)}$, $\left\{Z_{1}, Z_{2}, R_{1}, R_{2}, Q_{1 i}, Q_{2 i}, Q_{3 i}\right\} \in R^{n_{x} \times n_{x}}$, any appropriate dimensions matrices $\widetilde{S}, \widetilde{N}_{i} \in R^{\left(n_{x}+n_{f}+n_{w}\right) \times\left(n_{x}-r\right)}$, and satisfying
$\widetilde{E}^{T} \widetilde{S}=0, \quad \forall i, j \in I, \quad K:=\left[\begin{array}{ll}I_{n_{f}} & 0_{n_{f} \times\left(n_{x}-n_{f}\right)}\end{array}\right]^{T}$, the following linear matrix inequality sets are satisfied.

$$
\begin{aligned}
& \widetilde{\Omega}_{i}^{\prime}=\left[\begin{array}{ccccccc}
\widehat{\Omega}_{11} & \widehat{\Omega}_{12} & \widetilde{E}^{T} \Phi^{T} R_{1} \Phi \widetilde{E} & 0 & \widehat{\Omega}_{15} & \Omega_{16} & \widetilde{C}_{i}^{T} \\
* & \Omega_{22} & \widetilde{E}^{T} \Phi^{T} R_{2} \Phi \widetilde{E} & \widetilde{E}^{T} \Phi^{T} R_{2} \Phi \widetilde{E} & 0 & \Omega_{26} & \Phi^{T} \widetilde{C}_{d i}^{T} \\
* & * & \Omega_{33} & 0 & 0 & 0 & 0 \\
* & * & * & \Omega_{44} & 0 & 0 & 0 \\
* & * & * & * & -\gamma^{2} I & \Omega_{56} & \widetilde{D}_{i}^{T} \\
* & * & * & * & * & \Omega_{66} & 0 \\
* & * & * & * & * & * & -I
\end{array}\right]<0, \\
& \widehat{\widehat{\Omega}}_{11}=\left[\begin{array}{ccc}
\bar{\Omega}_{1 i} & \bar{\Omega}_{2 i} & \bar{\Omega}_{4 i} \\
* & \bar{\Omega}_{3 i} & 0 \\
* & * & \bar{\Omega}_{5 i}
\end{array}\right] \\
& \widehat{\Omega}_{12}=\left[\begin{array}{lll}
\Omega_{12}^{\prime T} & \Omega_{12}^{\prime \prime T} & \Omega_{12}^{\prime \prime \prime} T
\end{array}\right]^{T} \\
& \widehat{\Omega}_{15}=\left[\begin{array}{lll}
\Omega_{15}^{\prime T} & \Omega_{15}^{\prime \prime} T & \Omega_{15}^{\prime \prime \prime} T
\end{array}\right]^{T} \\
& \Omega_{12}^{\prime}=E^{T} P_{i(1)} A_{d i}+E^{T} K P_{i(2)} C_{d i}+N_{i} S^{T} A_{d i} \text {, } \\
& \Omega_{12}^{\prime \prime}=P_{i(2)}^{T} K^{T} A_{d i}+P_{i(2)} B_{F i} C_{d i} \text {, } \\
& \Omega_{12}^{\prime \prime \prime}=P_{i(4)}^{T} A_{d i} \\
& \Omega_{15}^{\prime}=\left[\begin{array}{c}
\left(N_{i} S^{T} B_{i}+E^{T} P_{i(1)} B_{i}\right)^{T} \\
\left(N_{i} S^{T} E_{i}+E^{T} P_{i(1)}^{T} E_{i}+E^{T} K P_{i(2)} B_{F i} D_{i}\right)^{T} \\
\left(E^{T} P_{i(1)} F_{i}+E^{T} K P_{i(2)} B_{F i} H_{i}+E^{T} P_{i(4)} B_{w f i}\right)^{T}
\end{array}\right]^{T}, \\
& \Omega_{15}^{\prime \prime}=\left[\begin{array}{lll}
P_{i(2)}^{T} K^{T} B_{i} & P_{i(2)}^{T} K^{T} E_{i}+P_{i(2)} B_{F i} D_{i} P_{i(2)}^{T} K^{T} F_{i}+P_{i(2)} B_{F i} H_{i}
\end{array}\right], \\
& \Omega_{15}^{\prime \prime \prime}=\left[\begin{array}{lll}
P_{i(4)}^{T} B_{i} & P_{i(4)}^{T} E_{i} P_{i(4)}^{T} F_{i}+P_{i(5)} B_{w f i}
\end{array}\right] \text {, } \\
& \bar{\Omega}_{1 i}= \begin{cases}\bar{\Omega}_{1 i}^{\prime}+E^{T}\left[\Xi_{k}^{(1 i)}+\Xi_{u c}^{(1 i l)}-\left(\lambda_{k}^{(i)}+\lambda_{u c}^{(i l)}\right) P_{j(1)}\right] E, & \text { if } i \in S_{k}^{(i)} \cup S_{u c}^{(i)}, j \in S_{u k}^{(i)}, \\
\bar{\Omega}_{1 i}^{\prime}+E^{T}\left[\Xi_{k}^{(1 i)}+\Xi_{u c}^{(1 i l)}+\lambda_{b}^{i} P_{i(1)}-\left(\lambda_{b}^{i}+\lambda_{k}^{(i)}+\lambda_{u c}^{(i l)}\right) P_{j(1)}\right] E, & \text { if } i \in S_{u k}^{(i)}, j \in S_{u k}^{(i)},\end{cases} \\
& \bar{\Omega}_{1 i}^{\prime}=\operatorname{sym}\left(E^{T} P_{i(1)} A_{i}\right)+\operatorname{sym}\left(A_{i}^{T} S N_{i}^{T}\right)+\operatorname{sym}\left(E^{T} K \bar{B}_{F i} C_{i}\right)+Q_{1 i}+Q_{2 i}+Q_{3 i}+d_{1} Z_{1}+d_{2} Z_{2}-E^{T} R_{1} E, \\
& \bar{\Omega}_{2 i}= \begin{cases}\bar{\Omega}_{2 i}^{\prime}+E^{T}\left[\Xi_{k}^{(2 i)}+\Xi_{u c}^{(2 i l)}-\left(\lambda_{k}^{(i)}+\lambda_{u c}^{(i l)}\right) P_{j(2)}\right] E, & \text { if } i \in S_{k}^{(i)} \cup S_{u c}^{(i)}, j \in S_{u k}^{(i)}, \\
\bar{\Omega}_{2 i}^{\prime}+E^{T}\left[\Xi_{k}^{(2 i)}+\Xi_{u c}^{(2 i l)}+\lambda_{b}^{i} P_{i(2)}-\left(\lambda_{b}^{i}+\lambda_{k}^{(i)}+\lambda_{u c}^{(i l)}\right) P_{j(2)}\right] E, & \text { if } i \in S_{u k}^{(i)}, j \in S_{u k}^{(i)},\end{cases} \\
& \bar{\Omega}_{2 i}^{\prime}=E^{T} K \bar{A}_{F i}+A_{i}^{T} K P_{i(2)}+C_{i}^{T} \bar{B}_{F i}^{T} \text {, } \\
& \bar{\Omega}_{3 i}=\left\{\begin{array}{l}
\bar{\Omega}_{3 i}^{\prime}+\Xi_{k}^{(2 i)}+\Xi_{u c}^{(2 i l)}-\left(\lambda_{k}^{(i)}+\lambda_{u c}^{(i l)}\right) P_{j(2)}, \quad \text { if } i \in S_{k}^{(i)} \cup S_{u c}^{(i)}, j \in S_{u k}^{(i)}, \\
\bar{\Omega}_{3 i}^{\prime}+\Xi_{k}^{(2 i)}+\Xi_{u c}^{(2 i l)}+\lambda_{b}^{i} P_{i(2)}-\left(\lambda_{b}^{i}+\lambda_{k}^{(i)}+\lambda_{u c}^{(i l)}\right) P_{j(2)}, \quad \text { if } i \in S_{u k}^{(i)}, j \in S_{u k}^{(i)},
\end{array}\right. \\
& \bar{\Omega}_{3 i}^{\prime}=\bar{A}_{F i}+\bar{A}_{F i}^{T} \text {, } \\
& \bar{\Omega}_{4 i}=\left\{\begin{array}{l}
\bar{\Omega}_{4 i}^{\prime}+E^{T}\left[\Xi_{k}^{(4 i)}+\Xi_{u c}^{(4 i l)}-\left(\lambda_{k}^{(i)}+\lambda_{u c}^{(i l)}\right) P_{j(4)}\right] E, \quad \text { if } i \in S_{k}^{(i)} \cup S_{u c}^{(i)}, j \in S_{u k}^{(i)}, \\
\bar{\Omega}_{4 i}^{\prime}+E^{T}\left[\Xi_{k}^{(4 i)}+\Xi_{u c}^{(4 i l)}+\lambda_{b}^{i} P_{i(4)}-\left(\lambda_{b}^{i}+\lambda_{k}^{(i)}+\lambda_{u c}^{(i l)}\right) P_{j(4)}\right] E, \quad \text { if } i \in S_{u k}^{(i)}, j \in S_{u k}^{(i)},
\end{array}\right. \\
& \bar{\Omega}_{4 i}^{\prime}=E^{T} P_{i(4)} A_{w f i}+A_{i}^{T} P_{i(4)} \text {, } \\
& \bar{\Omega}_{5 i}=\left\{\begin{array}{l}
\bar{\Omega}_{5 i}^{\prime}+\Xi_{k}^{(5 i)}+\Xi_{u c}^{(5 i l)}-\left(\lambda_{k}^{(i)}+\lambda_{u c}^{(i l)}\right) P_{j(5)}, \quad \text { if } i \in S_{k}^{(i)} \cup S_{u c}^{(i)}, j \in S_{u k}^{(i)}, \\
\bar{\Omega}_{5 i}^{\prime}+\Xi_{k}^{(5 i)}+\Xi_{u c}^{(5 i l)}+\lambda_{b}^{i} P_{i(5)}-\left(\lambda_{b}^{i}+\lambda_{k}^{(i)}+\lambda_{u c}^{(i l)}\right) P_{j(5)}, \quad \text { if } i \in S_{u k}^{(i)}, j \in S_{u k}^{(i)},
\end{array}\right. \\
& \bar{\Omega}_{5 i}^{\prime}=P_{i(5)}^{T} A_{w f i}+A_{w f i} P_{i(5)} \text {, } \\
& \Xi_{k}^{(1 i)}=\sum_{j \in S_{k}^{(i)}} \lambda_{i j} P_{j(1)}, \Xi_{k}^{(2 i)}=\sum_{j \in S_{k}^{(i)}} \lambda_{i j} P_{j(2)}, \Xi_{k}^{(4 i)}=\sum_{j \in S_{k}^{(i)}} \lambda_{i j} P_{j(4)}, \Xi_{k}^{(5 i)}=\sum_{j \in S_{k}^{(i)}} \lambda_{i j} P_{j(5)} \text {, } \\
& \Xi_{u c}^{(1 i l)}=\sum_{j \in S_{u c}^{(i)}} \tilde{\lambda}_{i j}^{(l)} P_{j(1)}, \Xi_{u c}^{(2 i l)}=\sum_{j \in S_{u c}^{(i)}} \tilde{\lambda}_{i j}^{(l)} P_{j(2)}, \Xi_{u c}^{(4 i l)}=\sum_{j \in S_{u c}^{(i)}} \tilde{\lambda}_{i j}^{(l)} P_{j(4)}, \Xi_{u c}^{(5 i l)}=\sum_{j \in S_{u c}^{(i)}} \tilde{\lambda}_{i j}^{(l)} P_{j(5)} \text {. }
\end{aligned}
$$


The gain matrices $\left(A_{F i}, B_{F i}, C_{F i}, D_{F i}\right)$ of fault detection filter (7) of system (1) can be obtained, and its parameter matrices are expressed as follows:

$$
\begin{aligned}
A_{F i} & :=P_{i(2)}^{-1} \bar{A}_{F i}, \\
B_{F i} & :=P_{i(2)}^{-1} \bar{B}_{F i}, \\
C_{F i} & :=\bar{C}_{F i}, \\
D_{F i} & :=\bar{D}_{F i} .
\end{aligned}
$$

Proof. In order to design high-performance full-order and reduced-order FDFs that meets the requirements, the Lyapunov matrix $P_{i}$ in Theorem 1 has the following form:

$$
P_{i}:=\left[\begin{array}{ccc}
P_{i(1)} & K P_{i(2)} & P_{i(4)} \\
* & P_{i(3)} & 0 \\
* & * & P_{i(5)}
\end{array}\right],
$$

where $K:=\left[I_{n_{f}} 0_{n_{f} \times\left(n_{x}-n_{f}\right)}\right]^{T}, \quad P_{i(1)} \in R^{n_{x} \times n_{x}}, P_{i(2)} \in R^{n_{f} \times n_{f}}$, $P_{i(3)} \in R^{n_{f} \times n_{f}}, P_{i(4)} \in R^{n_{x} \times n_{w}}$, and $P_{i(5)} \in R^{n_{w} \times n_{w}}$.

By using diagonal matrices $\operatorname{diag}\left\{I_{n_{x}}, P_{i(2)} P_{i(3)}^{-1}, I_{n_{f}}\right\}$ and $\operatorname{diag}\left\{I_{n_{x}}, P_{i(3)}^{-T} P_{i(2)}^{T}, I_{n_{f}}\right\}$ to perform the congruent transformation of (52), we can get

$$
\begin{gathered}
{\left[\begin{array}{ccc}
I_{n_{x}} & 0 & 0 \\
* & P_{i(2)} P_{i(3)}^{-1} & 0 \\
* & * & I_{n_{f}}
\end{array}\right]\left[\begin{array}{ccc}
P_{i(1)} & K P_{i(2)} & P_{i(4)} \\
* & P_{i(3)} & 0 \\
* & * & P_{i(5)}
\end{array}\right]\left[\begin{array}{ccc}
I_{n_{x}} & 0 & 0 \\
* & P_{i(3)}^{-T} P_{i(2)}^{T} & 0 \\
* & * & I_{n_{f}}
\end{array}\right]} \\
=\left[\begin{array}{ccc}
P_{i(1)} & K P_{i(2)} P_{i(3)}^{-T} P_{i(2)}^{T} & P_{i(4)} \\
* & P_{i(3)} P_{i(3)}^{-T} P_{i(2)}^{T} & 0 \\
* & * & P_{i(5)}
\end{array}\right]=:\left[\begin{array}{ccc}
P_{i(1)} & K \bar{P}_{i(2)} & P_{i(4)} \\
* & \bar{P}_{i(2)} & 0 \\
* & * & P_{i(5)}
\end{array}\right] .
\end{gathered}
$$

Therefore, without loss of generality, Lyapunov matrix $P_{i}$ can be specified as the following general form:

$$
P_{i}:=\left[\begin{array}{ccc}
P_{i(1)} & K P_{i(2)} & P_{i(4)} \\
* & P_{i(2)} & 0 \\
* & * & P_{i(5)}
\end{array}\right] .
$$

The matrix variable $P_{i(2)}$ in (54) can be combined with the gain matrices $A_{F i}$ and $B_{F i}$ of the filter to form new matrices, as follows:

$$
\bar{A}_{F i}:=P_{i(2)} A_{F i}, \quad B_{F i}:=P_{i(2)} B_{F i} .
$$

Equation (50) can be obtained by substituting (54) into equation (39). The proof is completed.

Remark 5. Theorem 3 presents a high efficiency method for designing fault detection filter for polytopic uncertain
CTSMJSs with time-varying delays that full-order and reduced-order FDFs can be expressed in a unified formula. In order to illustrate the effectiveness of the proposed approach, the following example will be used to verification.

\section{Numerical Example}

In this section, two examples are used to illustrate the practicability and effectiveness of our results.

Example 1. Consider the DC motor driving a load that switching is driven by a continuous-time Markov process $\{r(t), t>0\}[40,41]$. Similarly, by neglecting the DC motor inductance $L_{m}$, let $i(t), v(t)$, and $\dot{\bar{w}}(t)$ represent the electric current, the voltage, and the speed of the shaft at time $t$, respectively. According to the basic electrical and mechanic laws, we have

$$
\left\{\begin{array}{l}
\dot{\bar{w}}(t)=-\frac{b_{i}}{J_{i}} \bar{w}(t)+\frac{K_{t}}{J_{i}} i(t), \\
v(t)=K_{\bar{w}} \bar{w}(t)+R i(t),
\end{array}\right.
$$

where $K_{t}, K_{\bar{w}}$, and $R$ denote the torque constant, the electromotive force, and the electric resistor, respectively. $J_{i}$ and $b_{i}$ are defined as $J_{i}=J_{m}+J_{c i} / n^{2}$ and $b_{i}=b_{m}+b_{c i} / n^{2}$, where $J_{c i}$ and $J_{m}$ represent the moments of the load and the motor, respectively, and $b_{m}$ and $b_{c i}$ stand for the damping ratios with gear ratio $n$. Set $x_{1}(t)=\bar{w}(t)$ and $x_{2}(t)=i(t)$, and system (56) is expressed as

$$
\left[\begin{array}{ll}
1 & 0 \\
0 & 0
\end{array}\right] \dot{x}(t)=\left[\begin{array}{cc}
-\frac{b_{i}}{J_{i}} & K_{t} \\
J_{i} \\
K_{\bar{w}} & R
\end{array}\right] x(t)+\left[\begin{array}{l}
0 \\
1
\end{array}\right] v(t) .
$$

To guarantee the system stability, in this paper, we use the state feedback controller $v(t)=K_{i} x(t)$ and value $K_{1}=$ $[-1.90832-2.7715]$ and $K_{2}=[-2.3635-2.8652]$ in $[39,40]$. Without loss of generality, as used in [41], it is assumed that time delay $d(t)=0.1+0.05 \sin 2 t$ and system (57) can be written as

$$
\begin{aligned}
{\left[\begin{array}{ll}
1 & 0 \\
0 & 0
\end{array}\right] \dot{x}(t)=} & \left(\left[\begin{array}{cc}
-\frac{b_{i}}{J_{i}} & K_{t} \\
J_{i} \\
K_{\bar{w}} & R
\end{array}\right]+\left[\begin{array}{l}
0 \\
1
\end{array}\right] K_{i}\right) x(t) \\
& +\left[\begin{array}{cc}
0 & 0 \\
0.5 & 0
\end{array}\right] x(t-d(t)) .
\end{aligned}
$$

To ensure the validity of the actual example, we will use the parameters in $[40,41]$, where $J_{m}=0.5 \mathrm{~kg} \cdot \mathrm{m}$, $J_{c 1}=50 \mathrm{~kg} \cdot \mathrm{m}, J_{c 2}=150 \mathrm{~kg} \cdot \mathrm{m}, b_{c 1}=100, b_{c 2}=240, R=1$, $b_{m}=1, \quad K_{t}=3 \mathrm{Nm} / \mathrm{A}, \quad K_{\bar{w}}=1 \mathrm{Vs} / \mathrm{rad}, \quad n=10, \quad$ and 
$\Pi=\left[\begin{array}{cc}-0.0193 & 0.0193 \\ 0.0307 & -0.0307\end{array}\right]$, and the following closed-loop system can be obtained:

$$
E \dot{x}(t)=A(r(t)) x(t)+A_{d}(r(t)) x(t-d(t)),
$$

where $\quad E=\left[\begin{array}{ll}1 & 0 \\ 0 & 0\end{array}\right], \quad A_{1}=\left[\begin{array}{cc}-2.0000 & 3.0000 \\ -0.9083 & -1.7715\end{array}\right]$, $A_{2}=\left[\begin{array}{cc}-1.7000 & 1.5000 \\ -1.3635 & -1.8652\end{array}\right]$, and $A_{d 1}=A_{d 2}=\left[\begin{array}{cc}0 & 0 \\ 0.5 & 0\end{array}\right]$.

In order to design the full-order and reduced-order FDFs about system (59), the other parameters in [41] are given as

$$
\begin{aligned}
& B_{1}=[-2.5 ;-0.3] \text {, } \\
& B_{2}=[1.5 ;-0.2] \text {, } \\
& E_{1}=[-0.2 ;-0.1] \text {, } \\
& E_{2}=[0.15 ;-0.12] \text {, } \\
& F_{1}=[-0.5 ; 0.4] \text {, } \\
& F_{2}=[0.6 ; 0.3] \text {, } \\
& C_{1}=\left[\begin{array}{ll}
-0.5 & 1.2
\end{array}\right] \text {, } \\
& C_{2}=\left[\begin{array}{ll}
-0.35 & 0.7
\end{array}\right] \text {, } \\
& C_{d 1}=\left[\begin{array}{ll}
-0.5 & -1.6
\end{array}\right] \text {, } \\
& C_{d 2}=\left[\begin{array}{ll}
-0.36 & 0.8
\end{array}\right] \text {, } \\
& D_{1}=1.2 \text {, } \\
& D_{2}=0.25 \text {, } \\
& H_{1}=2 \text {, } \\
& H_{2}=-0.5 \text {, } \\
& A_{w f i}=-5 \text {, } \\
& B_{w f i}=3 \text {, } \\
& C_{w f i}=1 \text {, } \\
& D_{w f i}=0 .
\end{aligned}
$$

By Theorem 3, lettingS $=[0 ; 1]$ and $\gamma=2$, we can get the parameters of the desired full-order and reduced-order FDFs, which are given as follows.

(1) Parameter matrices of full-order FDF:

$$
\begin{aligned}
& A_{F 1}=\left[\begin{array}{cc}
-0.8590 & 0.2076 \\
-0.0525 & -1.3138
\end{array}\right] \text {, } \\
& B_{F 1}=[0.2362 ; 0.0058] \text {, } \\
& C_{F 1}=\left[\begin{array}{ll}
-0.0602 & 0.0866
\end{array}\right] \text {, } \\
& D_{F 1}=[0.1134] \text {, } \\
& A_{F 2}=\left[\begin{array}{cc}
-0.8447 & 0.7543 \\
-0.5808 & -1.3574
\end{array}\right] \text {, } \\
& B_{F 2}=[0.1068 ; 0.4773] \text {, } \\
& C_{F 2}=\left[\begin{array}{ll}
-0.0152 & 0.0299
\end{array}\right] \text {, } \\
& D_{F 2}=[-0.3351] \text {. }
\end{aligned}
$$

(2) Parameter matrices of reduced-order FDF:

$$
\begin{aligned}
& A_{F 1}=[-4.9187], \\
& B_{F 1}=[1.3578], \\
& C_{F 1}=[-0.0714], \\
& D_{F 1}=[0.1160], \\
& A_{F 2}=[-2.6482], \\
& B_{F 2}=[0.3304], \\
& C_{F 2}=[-0.0165], \\
& D_{F 2}=[-0.3374] .
\end{aligned}
$$

Remark 6. Compared with [41], the proposed approach in this paper can not only solve the parameters of full-order FDF for DC motor but also figure out the parameters of reduced-order FDF. However, when the DC motor driving a load that switching is driven to start with the TRs have unknown elements and many uncertain elements, the proposed method is also practical. The following examples will further illustrate the effectiveness of the proposed method for FDFs design for different TRMs. We compare and analyse the $H_{\infty}$ performance index $\gamma_{\min }$ and the number of decision variables of full-order and reduced-order FDF for four different TRMs.

Example 2. In this section, we select a system with four modals to verify the effectiveness of the designed full-order and reduced-order FDFs of CTSMJSs with time-varying delays and four different TRMs. The parameters matrices can be described as follows: 


$$
\begin{aligned}
& A_{1}=\left[\begin{array}{cc}
-2.8 & 0.4 \\
-3.1 & -1.6
\end{array}\right] \text {, } \\
& A_{2}=\left[\begin{array}{cc}
2.5 & 0.5 \\
-0.6 & -1.0
\end{array}\right] \text {, } \\
& A_{3}=\left[\begin{array}{cc}
-2.5 & -0.5 \\
0.2 & -1.8
\end{array}\right] \text {, } \\
& A_{4}=\left[\begin{array}{cc}
-1.5 & 0 \\
0 & -0.9
\end{array}\right] \text {, } \\
& A_{d 1}=\left[\begin{array}{cc}
0.1 & -0.2 \\
-0.1 & -0.3
\end{array}\right] \text {, } \\
& A_{d 2}=\left[\begin{array}{cc}
0.1 & 0.2 \\
0 & 0.1
\end{array}\right] \text {, } \\
& A_{d 3}=\left[\begin{array}{cc}
0.1 & -0.2 \\
-0.1 & -0.3
\end{array}\right] \text {, } \\
& A_{d 4}=\left[\begin{array}{cc}
0.1 & -0.2 \\
-0.1 & -0.1
\end{array}\right] \text {, } \\
& B_{1}=[0.6 ; 0] \text {, } \\
& B_{2}=[0.4 ;-0.2] \text {, } \\
& B_{3}=[0.3 ; 0.8] \text {, } \\
& B_{4}=[0.3 ;-0.2] \text {, } \\
& C_{1}=\left[\begin{array}{ll}
0.5 & 0.3
\end{array}\right], \\
& C_{2}=\left[\begin{array}{ll}
0.4 & -0.5
\end{array}\right] \text {, } \\
& C_{3}=\left[\begin{array}{ll}
-0.3 & -0.4
\end{array}\right] \text {, } \\
& C_{4}=\left[\begin{array}{ll}
0.5 & -0.2
\end{array}\right], \\
& C_{d 1}=\left[\begin{array}{ll}
0.1 & -0.3
\end{array}\right] \text {, } \\
& C_{d 2}=\left[\begin{array}{ll}
0.2 & -0.3
\end{array}\right] \text {, } \\
& C_{d 3}=\left[\begin{array}{ll}
0.3 & -0.4
\end{array}\right] \text {, } \\
& C_{d 4}=\left[\begin{array}{ll}
0.5 & -0.1
\end{array}\right] \text {, } \\
& D_{1}=0.5 \text {, } \\
& D_{2}=0.3 \text {, } \\
& D_{3}=-0.3 \text {, } \\
& D_{4}=0.5 \text {, } \\
& E_{1}=[0.4 ;-0.3] \text {, } \\
& E_{2}=[0 ;-0.3] \text {, } \\
& E_{3}=[-0.3 ; 0.1] \text {, } \\
& E_{4}=[-0.5 ; 0], \\
& F_{1}=[0.2 ; 0] \text {, } \\
& F_{2}=[0.1 ;-0.3] \text {, } \\
& F_{3}=[0.3 ;-0.2] \text {, } \\
& F_{4}=[0.2 ;-0.1] \text {, } \\
& H_{1}=0.2 \text {, } \\
& H_{2}=-0.2 \text {, } \\
& H_{3}=0.3 \text {, } \\
& H_{4}=-0.4 \text {, } \\
& E=\left[\begin{array}{llll}
1 & 0 ; & 0 & 0
\end{array}\right] \text {, } \\
& S=[0 ; 1], \\
& A_{w f i}=-5 \text {, } \\
& B_{w f i}=3 \text {, } \\
& C_{w f i}=1 \text {, } \\
& D_{w f i}=0 \text {. }
\end{aligned}
$$

To make the convenience of simulation, the weighted fault frequency function and known control input signal to be set as $W_{f}(s)=3 / s+5$ and $u(t)=0.1+0.05 \cos (t)$, the Gaussian white noise signal with amplitude less than 0.2 is selected as the exogenous disturbance input $w(t)$, respectively. The range of time-varying delay $d(t)$ satisfies $0.1 \leq d(t) \leq 0.6$ and $\dot{d}(t) \leq 0.3$ for $0 \leq t \leq 60$. In this paper, the simulation example selects the fault expression $f(t)$ that was used in many classic papers as the potential fault signal [31-34]. The fault detection graph simulated by this fault expression is not only simple but also clear; we can clearly see whether the fault detection filter is effective or not. The fault signal $f(t)$ is

$$
f(t)=\left\{\begin{array}{l}
1.5, \quad 20 \leq t \leq 40, \\
0, \quad \text { others. }
\end{array}\right.
$$

Table 1 lists the four different cases TRMs, and Figure 1 shows the simulation results of four different cases Markov chain $r(t)$ evolution processes.

For Case 2, all the rows remain the same except for the third row, it contains two uncertain TRs, which means the TRM includes two vertices $\Lambda_{3(s)}$ and $s=1,2$ in third rows, and the TRM in the third row can be expressed as

$$
\Lambda_{3(1)}=\left[\begin{array}{llll}
0.4 & \hat{\lambda}_{32} & -1.3 & \hat{\lambda}_{34}
\end{array}\right], \quad \Lambda_{3(2)}=\left[\begin{array}{llll}
0.6 & \hat{\lambda}_{32} & -1.1 & \hat{\lambda}_{34}
\end{array}\right] \text {. }
$$

At the same time, we set the lower bounds $\widehat{\lambda}_{2 b(44)}=-1$ of unknown diagonal elements $\widehat{\lambda}_{44}$ in Case 2; set $\widehat{\lambda}_{3 b(44)}=-1$ in Case $3 ;$ and assign $\hat{\lambda}_{4 b(11)}=-0.8, \quad \hat{\lambda}_{4 b(22)}=-1.2$, $\widehat{\lambda}_{4 b(33)}=-1.3$, and $\widehat{\lambda}_{4 b(44)}=-0.7$ in Case 4 , respectively.

When the system parameters are presented, the linear matrix inequalities in Theorem 3 can be solved by MATLAB, and the full-order and reduced-order FDFs parameter matrices for four different cases of TRMs can be obtained as follows.

Case 1. TRM is completely known.

(1) Parameter matrices of full-order FDF with completely known TRM:

$$
\begin{aligned}
& A_{F 1}=\left[\begin{array}{cc}
-2.3922 & 0.1886 \\
0.3139 & -3.5091
\end{array}\right], \\
& B_{F 1}=[-0.8997 ; 1.2641] \text {, } \\
& C_{F 1}=\left[\begin{array}{ll}
-0.1449 & 0.1438
\end{array}\right] \text {, } \\
& D_{F 1}=[0.1801] \text {, } \\
& A_{F 2}=\left[\begin{array}{cc}
-2.3114 & 2.0323 \\
-1.7332 & -2.7174
\end{array}\right] \text {, } \\
& B_{F 2}=[0.5977 ;-0.7239] \text {, } \\
& C_{F 2}=\left[\begin{array}{ll}
-0.0860 & -0.1613
\end{array}\right] \text {, } \\
& D_{F 2}=[-0.0504] \text {, } \\
& A_{F 3}=\left[\begin{array}{cc}
-2.3830 & -0.3480 \\
0.1603 & -3.8508
\end{array}\right] \text {, }
\end{aligned}
$$




$$
\begin{aligned}
& B_{F 3}=[-1.3546 ;-0.6935], \\
& C_{F 3}=\left[\begin{array}{ll}
-0.1584 & -0.1482
\end{array}\right], \\
& D_{F 3}=[0.2610], \\
& A_{F 4}=\left[\begin{array}{cc}
-1.4610 & 0.0310 \\
-0.0857 & -2.3989
\end{array}\right], \\
& B_{F 4}=[1.5553 ;-0.4436], \\
& C_{F 4}=[-0.2164-0.0215], \\
& D_{F 4}=[-0.2857] .
\end{aligned}
$$

(2) Parameter matrices of reduced-order FDF with completely known TRM:

$$
\begin{aligned}
& A_{F 1}=[-2.8377], \\
& B_{F 1}=[-1.0126], \\
& C_{F 1}=[-0.1645], \\
& D_{F 1}=[0.1884], \\
& A_{F 2}=[-2.6302], \\
& B_{F 2}=[0.4328], \\
& C_{F 2}=[-0.0973], \\
& D_{F 2}=[-0.0482], \\
& A_{F 3}=[-2.6505], \\
& B_{F 3}=[-1.2617], \\
& C_{F 3}=[-0.2041], \\
& D_{F 3}=[0.2795], \\
& A_{F 4}=[-0.9532], \\
& B_{F 4}=[1.0858], \\
& C_{F 4}=[-0.2087], \\
& D_{F 4}=[-0.3146],
\end{aligned}
$$

$$
\begin{aligned}
& A_{F 1}=\left[\begin{array}{cc}
-1.0126 & 0.0893 \\
-0.0672 & -1.8273
\end{array}\right], \\
& B_{F 1}=[-0.0499 ; 0.8281], \\
& C_{F 1}=\left[\begin{array}{ll}
-0.1424 & 0.0691
\end{array}\right], \\
& D_{F 1}=[0.0739], \\
& A_{F 2}=\left[\begin{array}{ll}
-0.9554 & 0.4547 \\
-0.4769 & -1.2196
\end{array}\right], \\
& B_{F 2}=\left[\begin{array}{ll}
-0.0562 ; 0.4387 &
\end{array},\right. \\
& C_{F 2}=\left[\begin{array}{ll}
-0.0964 & -0.0333
\end{array}\right], \\
& D_{F 2}=[0.0367], \\
& A_{F 3}=\left[\begin{array}{cc}
-1.1803 & -0.0917 \\
0.0210 & -1.8310
\end{array}\right], \\
& B_{F 3}=\left[\begin{array}{ll}
-0.6052 ;-0.3351
\end{array}\right], \\
& C_{F 3}=\left[\begin{array}{ll}
-0.0943 & -0.0407
\end{array}\right], \\
& D_{F 3}=[0.1409], \\
& A_{F 4}=\left[\begin{array}{ll}
-0.9007 & 0.1558 \\
-0.1743 & -2.3419
\end{array}\right], \\
& B_{F 4}=[-0.2081 ;-0.1826], \\
& C_{F 4}=\left[\begin{array}{ll}
-0.1404 & 0.0017
\end{array}\right], \\
& D_{F 4}=[-0.0472] .
\end{aligned}
$$

(2) Parameter matrices of reduced-order FDF with polytopic uncertain TRM:

$$
\begin{aligned}
A_{F 1} & =[-4.1974], \\
B_{F 1} & =[0.1968], \\
C_{F 1} & =[-0.1456], \\
D_{F 1} & =[0.579], \\
A_{F 2} & =[-3.8601], \\
B_{F 2} & =[0.2387], \\
C_{F 2} & =[-0.1003], \\
D_{F 2} & =[0.0377], \\
A_{F 3} & =[-4.5124], \\
B_{F 3} & =[0.1336], \\
C_{F 3} & =[-0.0944], \\
D_{F 3} & =[0.1374], \\
A_{F 4} & =[-0.9109], \\
B_{F 4} & =[-0.2188], \\
C_{F 4} & =[-0.1296], \\
D_{F 4} & =[-0.0409] .
\end{aligned}
$$

Case 3. TRM is partly unknown.

(1) Parameter matrices of full-order FDF with partly unknown TRM:
(1) Parameter matrices of full-order FDF with polytopic uncertain TRM:

Case 2. TRM is polytopic uncertain. 
TABLE 1: TRMs of four different cases.

Case 1: completely known TRM Case 2: polytopic uncertain TRM Case 3: partly unknown TRM Case 4: completely unknown TRM

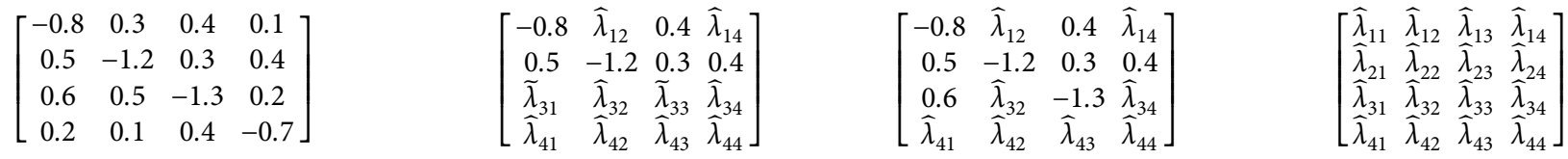

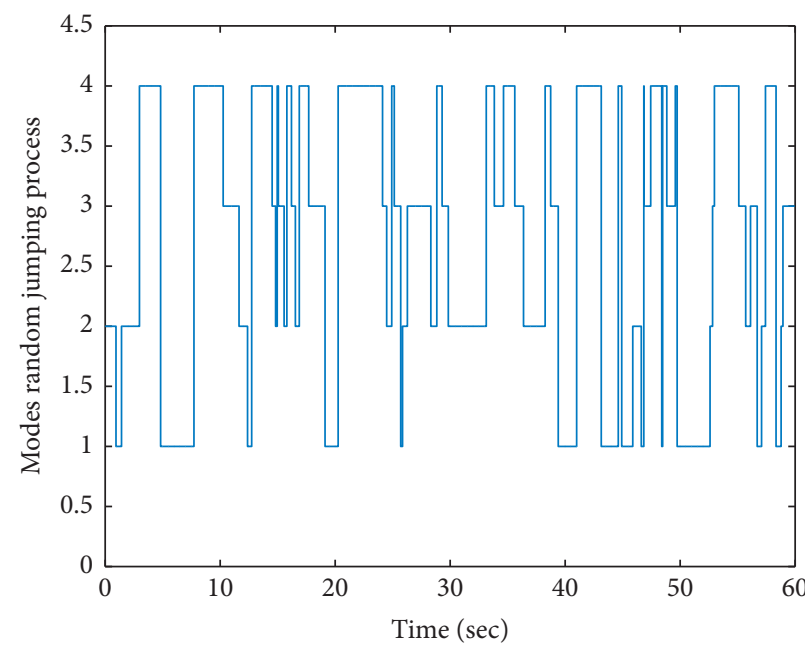

(a)

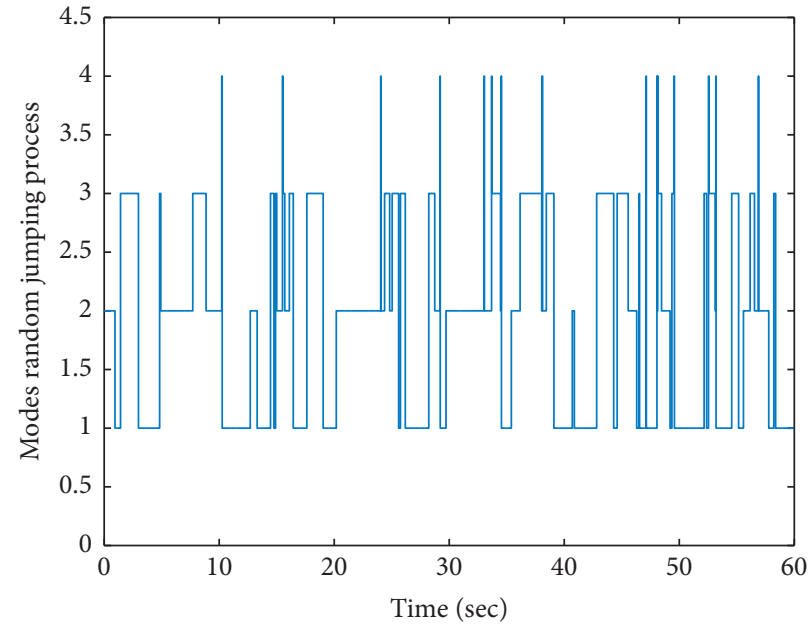

(c)

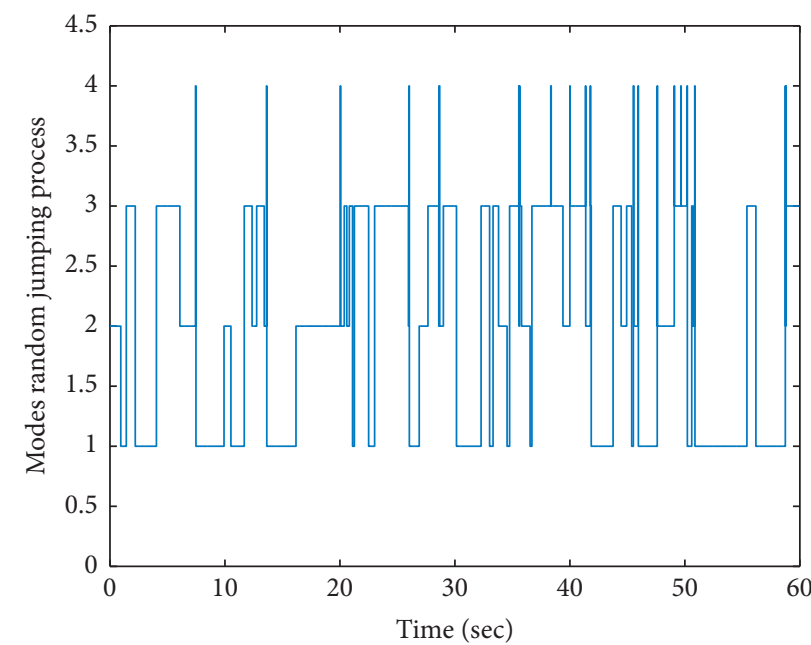

(b)

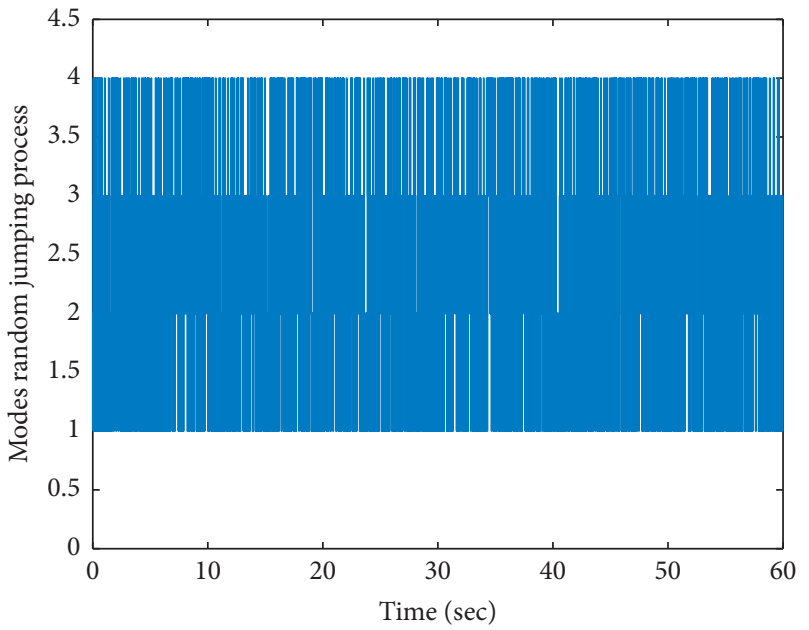

(d)

FIgure 1: The modes random jumping process of four different TRMs cases. (a) Case 1. TRM is completely known. (b) Case 1. TRM is polytopic uncertain. (c) TRM is partly unknown. (d) TRM is completely unknown. 


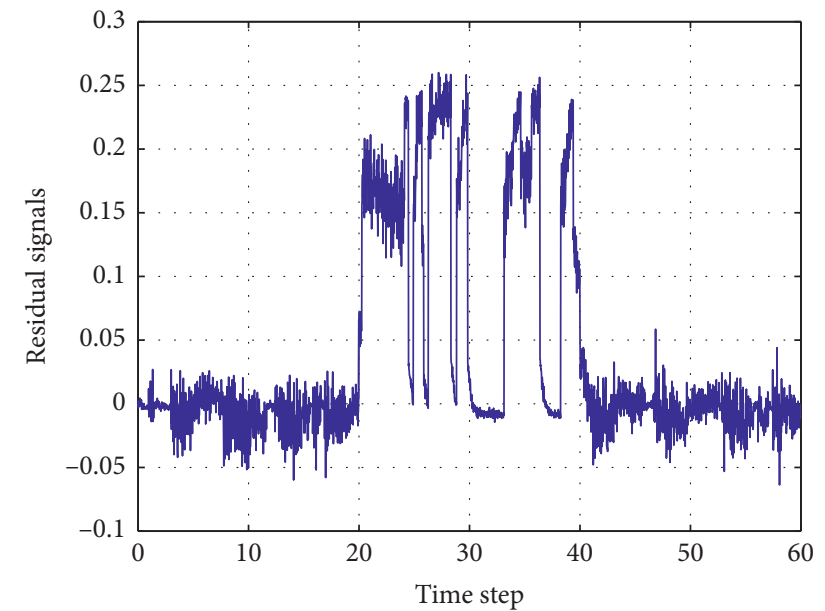

(a)

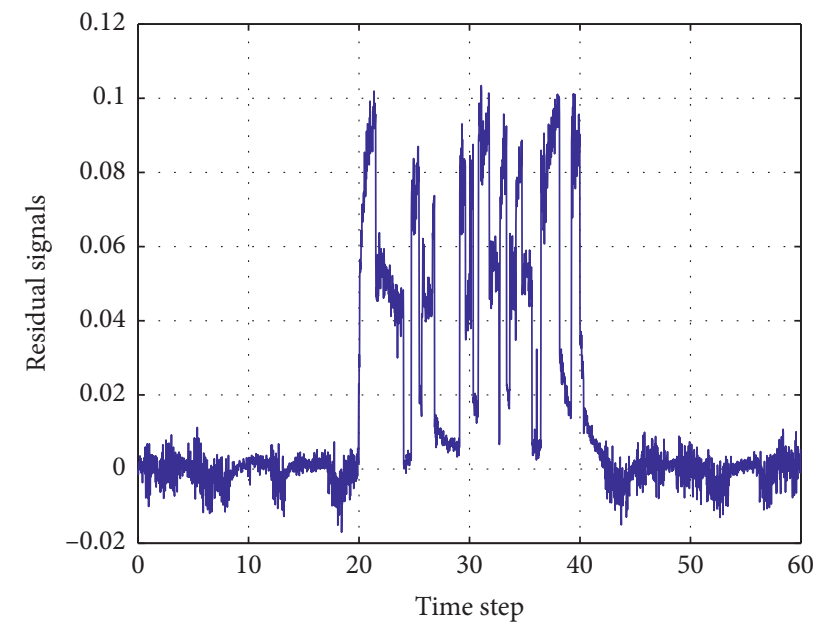

(c)

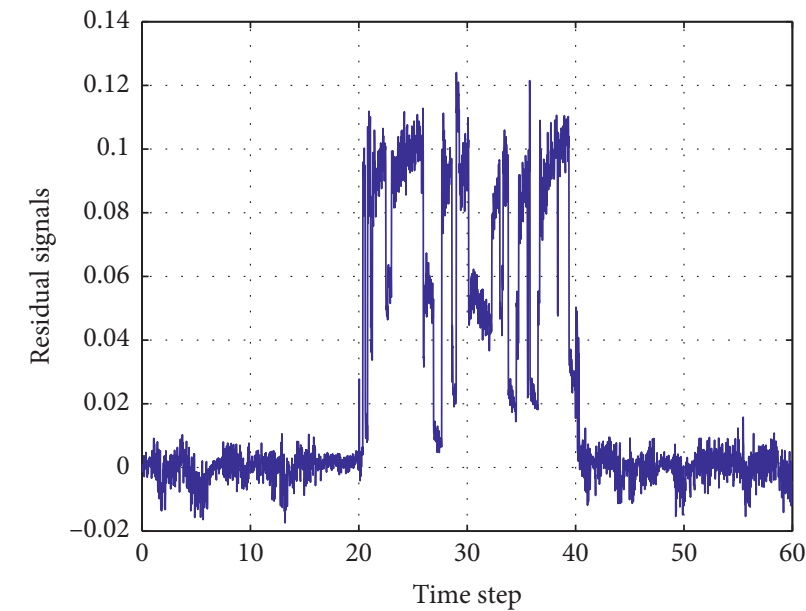

(b)

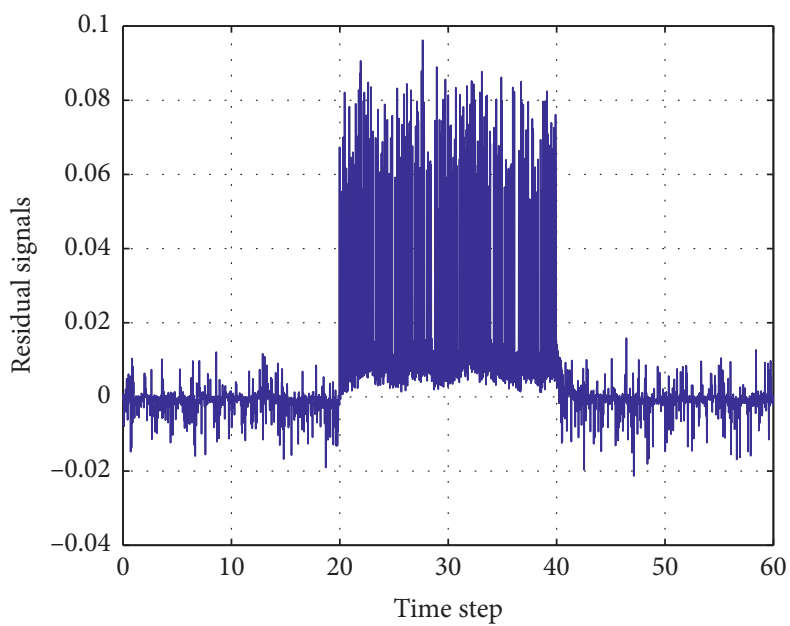

(d)

FIgURe 2: Residual generation of full-order FDF for four different TRMs cases. (a) Case 1. TRM is completely known. (b) Case 1. TRM is polytopic uncertain. (c) TRM is partly unknown. (d) TRM is completely unknown.

$$
\begin{aligned}
& A_{F 1}=\left[\begin{array}{cc}
-1.0078 & 0.0714 \\
-0.0417 & -1.8167
\end{array}\right] \text {, } \\
& B_{F 1}=[-0.0856 ; 0.8378] \text {, } \\
& C_{F 1}=\left[\begin{array}{ll}
-0.1293 & 0.0672
\end{array}\right] \text {, } \\
& D_{F 1}=\text { [0.0757], } \\
& A_{F 2}=\left[\begin{array}{cc}
-0.9593 & 0.4838 \\
-0.4977 & -1.2134
\end{array}\right] \text {, } \\
& B_{F 2}=[0.0874 ;-0.4544] \text {, } \\
& C_{F 2}=\left[\begin{array}{ll}
-0.0874 & -0.0316
\end{array}\right] \text {, } \\
& D_{F 2}=[0.0309] \text {, } \\
& A_{F 3}=\left[\begin{array}{cc}
-1.0948 & -0.0532 \\
0.0106 & -1.7874
\end{array}\right] \text {, } \\
& B_{F 3}=[-0.6223 ;-0.3788] \text {, } \\
& C_{F 3}=\left[\begin{array}{ll}
-0.1368 & -0.0554
\end{array}\right] \text {, } \\
& D_{F 3}=[0.1112] \text {, } \\
& A_{F 4}=\left[\begin{array}{cc}
-0.9441 & 0.1718 \\
-0.1679 & -2.3823
\end{array}\right] \text {, } \\
& B_{F 4}=[-0.1383 ;-0.1917] \text {, } \\
& C_{F 4}=\left[\begin{array}{ll}
-0.1312 & -0.0015
\end{array}\right] \text {, } \\
& D_{F 4}=[-0.0585] \text {. }
\end{aligned}
$$

(2) Parameter matrices of reduced-order FDF with partly unknown TRM:

$$
\begin{aligned}
A_{F 1} & =[-3.2699], \\
B_{F 1} & =[-0.0283], \\
C_{F 1} & =[-0.1212], \\
D_{F 1} & =[0.0680], \\
A_{F 2} & =[-3.9731], \\
B_{F 2} & =[0.3550], \\
C_{F 2} & =[-0.0852], \\
D_{F 2} & =[-0.0278], \\
A_{F 3} & =[-3.8903], \\
B_{F 3} & =[-0.1837], \\
C_{F 3} & =[-0.1315], \\
D_{F 3} & =[0.1106], \\
A_{F 4} & =[-0.9442], \\
B_{F 4} & =[-0.0697], \\
C_{F 4} & =[-0.1344], \\
D_{F 4} & =[-0.0503] .
\end{aligned}
$$

Case 4. TRM is completely unknown.

(1) Parameter matrices of full-order FDF with completely unknown TRM: 


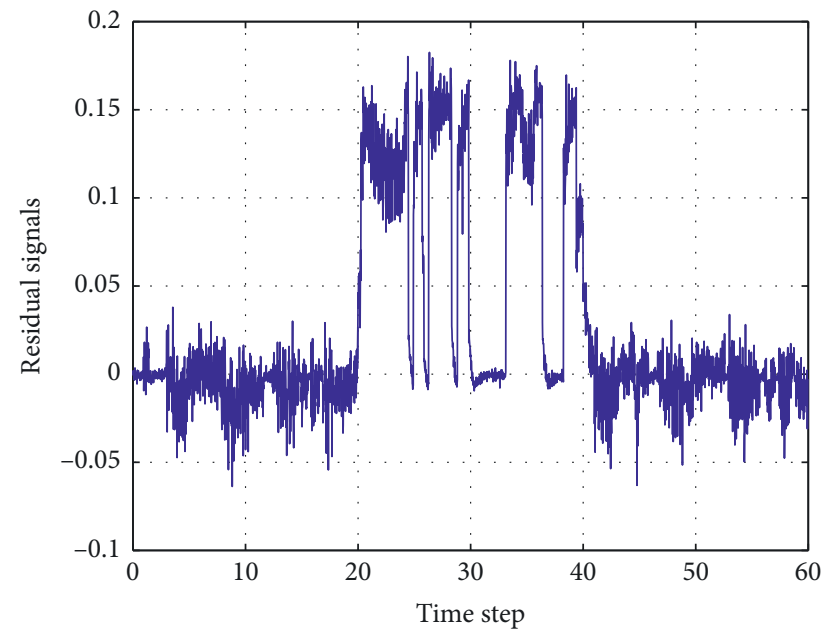

(a)

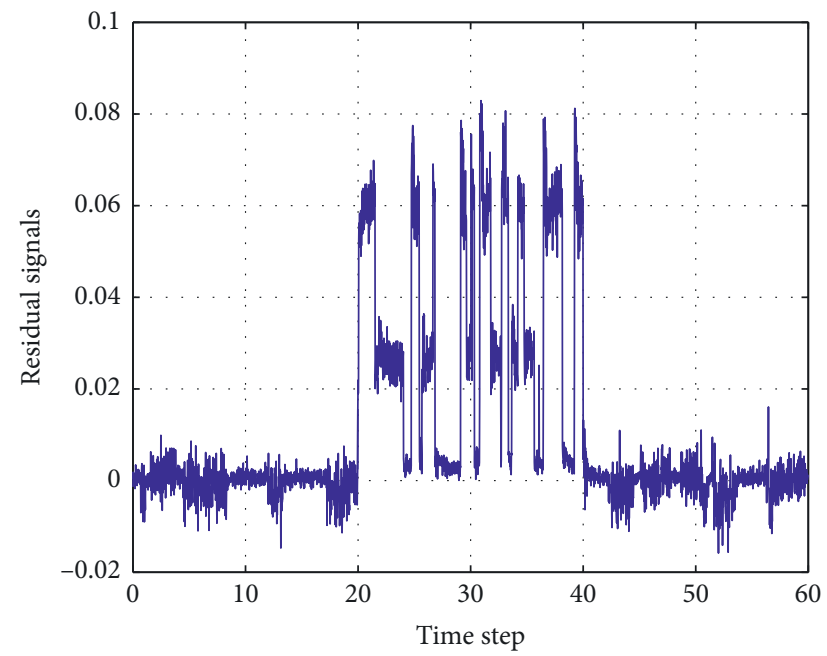

(c)

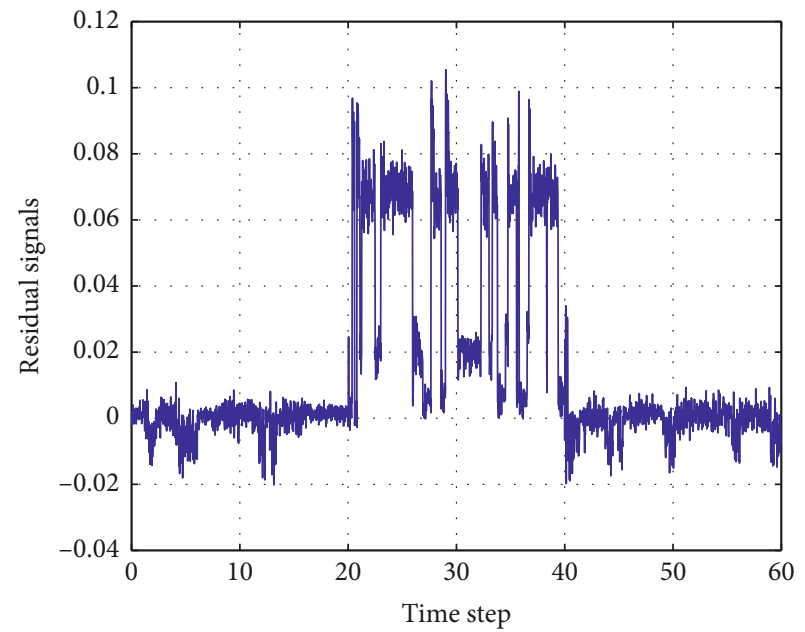

(b)

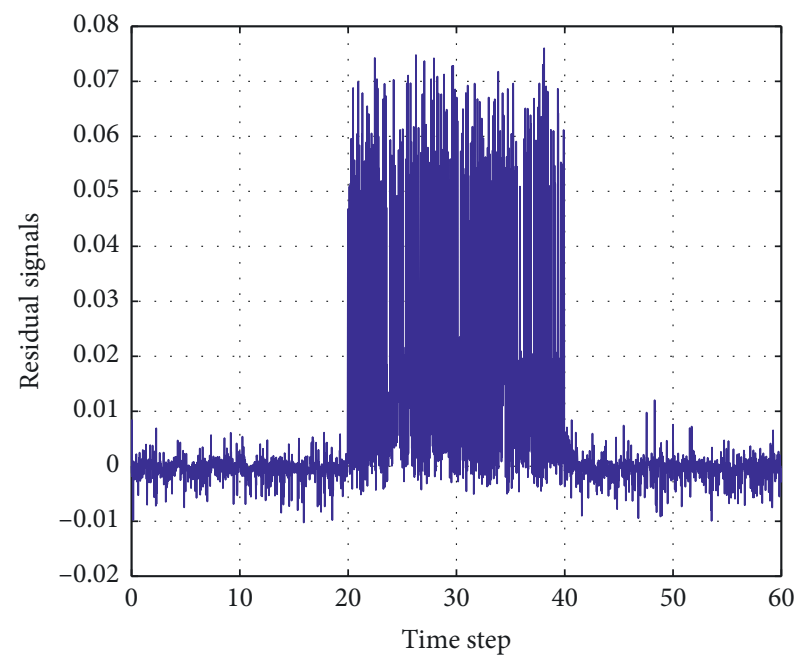

(d)

FIGURE 3: Residual generation of reduced-order FDF for four different TRMs cases. (a) Case 1. TRM is completely known. (b) Case 1. TRM is polytopic uncertain. (c) TRM is partly unknown. (d) TRM is completely unknown.

$$
\begin{aligned}
& A_{F 1}=\left[\begin{array}{cc}
-1.2535 & -0.2273 \\
0.3095 & -3.1673
\end{array}\right], \\
& B_{F 1}=[-0.3551 ; 0.1637] \text {, } \\
& C_{F 1}=\left[\begin{array}{ll}
-0.1840 & 0.0571
\end{array}\right] \text {, } \\
& D_{F 1}=[0.0036] \text {, } \\
& A_{F 2}=\left[\begin{array}{cc}
-1.3733 & 0.1410 \\
-0.1867 & -3.1945
\end{array}\right] \text {, } \\
& B_{F 2}=[-0.0225 ;-0.2367] \text {, } \\
& C_{F 2}=\left[\begin{array}{ll}
-0.0935 & -0.0711
\end{array}\right] \text {, } \\
& D_{F 2}=[-0.0171] \text {, } \\
& A_{F 3}=\left[\begin{array}{cc}
-1.7144 & 0.1364 \\
-0.3325 & -3.6592
\end{array}\right] \text {, } \\
& B_{F 3}=[-1.0059 ;-0.3267] \text {, } \\
& C_{F 3}=\left[\begin{array}{ll}
-0.0368 & -0.0623
\end{array}\right] \text {, } \\
& D_{F 3}=[0.1701] \text {, } \\
& A_{F 4}=\left[\begin{array}{cc}
-0.9380 & 0.1069 \\
-0.1388 & -2.4802
\end{array}\right] \text {, } \\
& B_{F 4}=[-0.6428 ;-0.0735] \text {, } \\
& C_{F 4}=\left[\begin{array}{ll}
0.1001 & -0.0100
\end{array}\right] \text {, } \\
& D_{F 4}=[-0.0231] \text {. }
\end{aligned}
$$

(2) Parameter matrices of reduced-order FDF with completely unknown TRM:

$$
\begin{aligned}
A_{F 1} & =[-3.6041], \\
B_{F 1} & =[-0.9925], \\
C_{F 1} & =[-0.1896], \\
D_{F 1} & =[0.0061], \\
A_{F 2} & =[-2.7577], \\
B_{F 2} & =[-0.1842], \\
C_{F 2} & =[-0.1007], \\
D_{F 2} & =[-0.0224], \\
A_{F 3} & =[-3.6929], \\
B_{F 3} & =[-0.2183], \\
C_{F 3} & =[-0.0513], \\
D_{F 3} & =[0.0967], \\
A_{F 4} & =[-1.2794], \\
B_{F 4} & =[-0.9063], \\
C_{F 4} & =[-0.1050], \\
D_{F 4} & =[-0.0387] .
\end{aligned}
$$




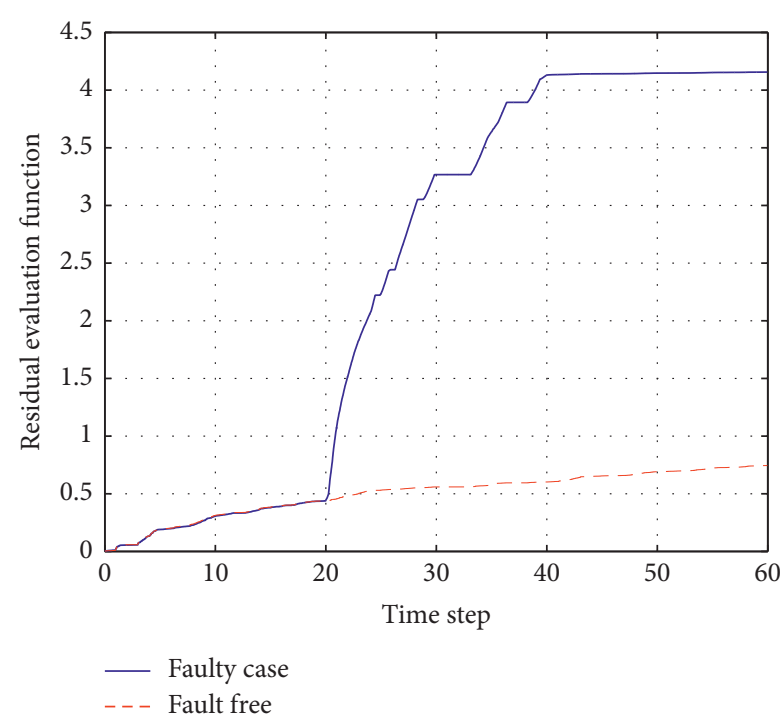

(a)

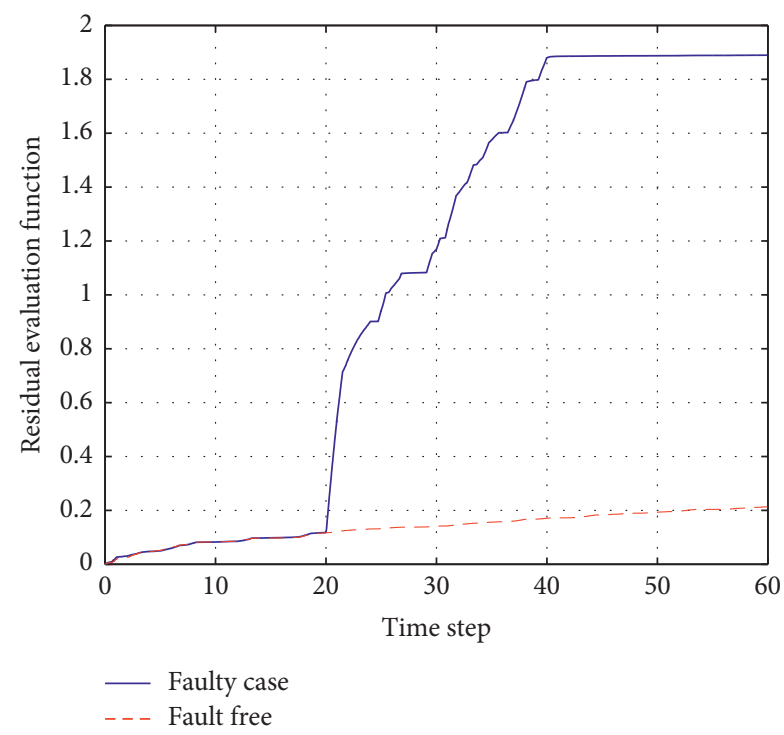

(c)

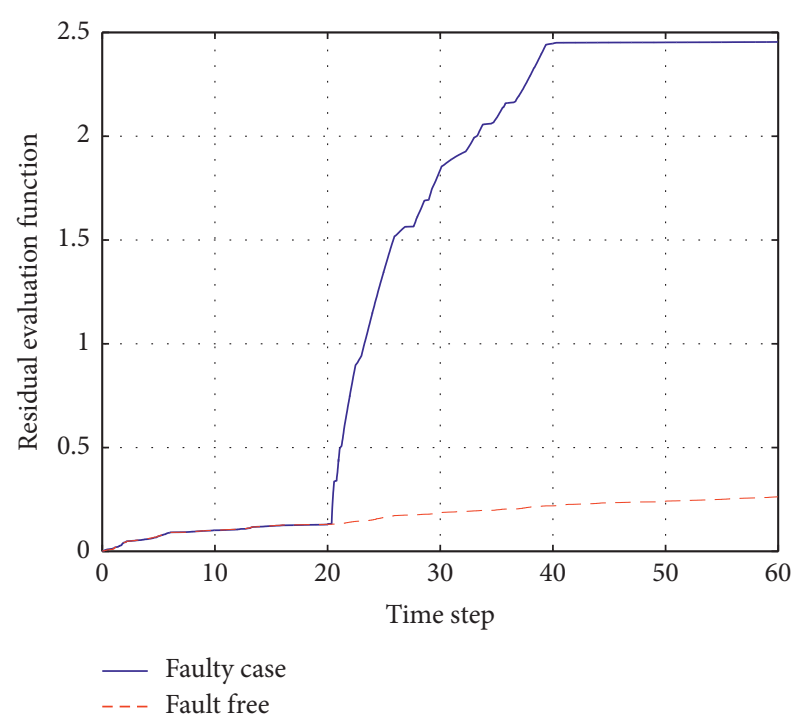

(b)

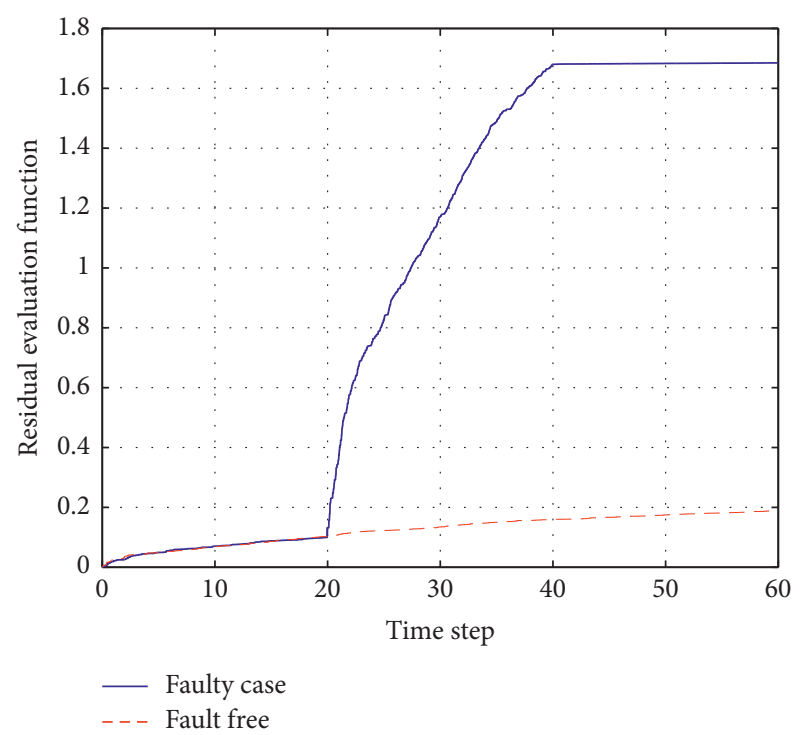

(d)

FIGURE 4: Residual evaluation function curves of full-order FDF for four different TRMs cases. (a) Case 1. TRM is completely known. (b) Case 1. TRM is polytopic uncertain. (c) TRM is partly unknown. (d) TRM is completely unknown.

Remark 7. According to Theorem 3 and the simulation results, we can obtain that the selection dimension of $P_{i(2)} \in R^{n_{f} \times n_{f}}$ is the key to obtain the parameters of the fullorder and reduced-order FDFs. When $n_{f}=n_{x}$, the parameters of the full-order FDF can be determined. When $n_{f}<n_{x}$, the parameters of the reduced-order FDF can be determined. For simplicity, the parameter matrices selected in this paper is two-dimensional, so only one-dimensional reduced-order FDF parameters are simulated. The method for determining the parameters of the reduced-order FDF is suitable for multidimensional matrices. By adjusting the dimensions of $P_{i(2)}$, the parameters of the reduced-order FDF of different dimensions can be obtained. For example, when the parameter matrices of the full-order fault detection filter are three-dimensional, the one-dimensional and two- dimensional parameter matrices of reduced-order FDFs can be obtained, respectively.

For convenience of simulation, the shortest time step $L_{s_{\min }}$ is set as $J(\widehat{r}(t))>J_{t h}, N_{D V}$ represents the number of decision variables, where $J_{t h}=\sup _{0 \neq w \in l_{2}, 0 \neq u_{k} \in l_{2}, f=0} E\left[\sqrt{\int_{k_{0}}^{k_{0}+L} \widehat{r}^{T}(t) \widehat{r}(t) d t}\right]$, and the minimum $H_{\infty}$ performance is $\gamma_{\min }$. Next, we will illustrate the effectiveness of the full-order and reduced-order FDFs for four diffident cases of TRM by simulation analysis and parameter comparison lists.

Figures 2 and 3 demonstrate residual generation of fullorder and reduced-order FDFs for four different TRMs cases, respectively. Obviously, it can be seen by residual 


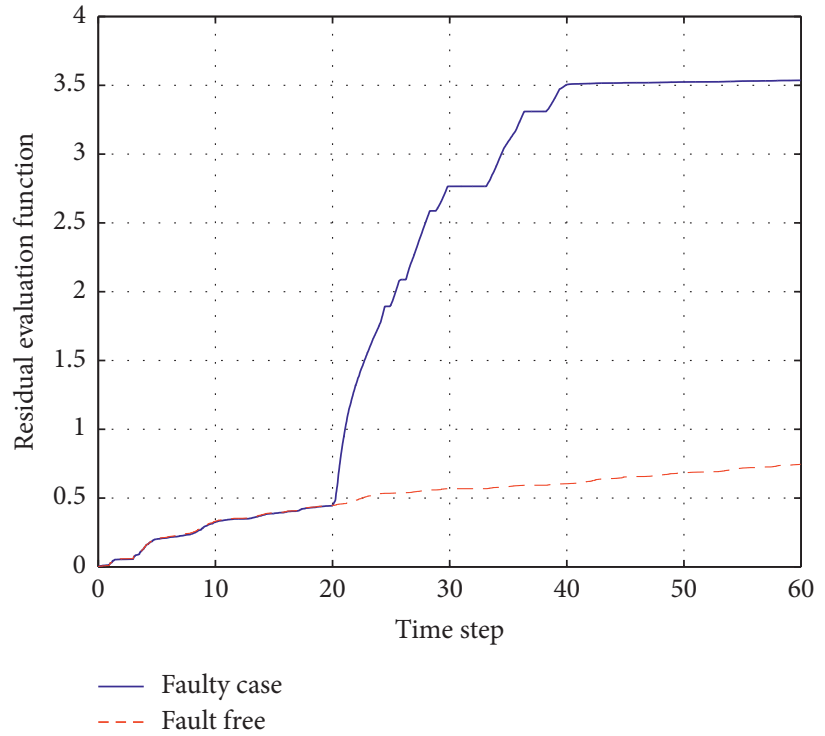

(a)

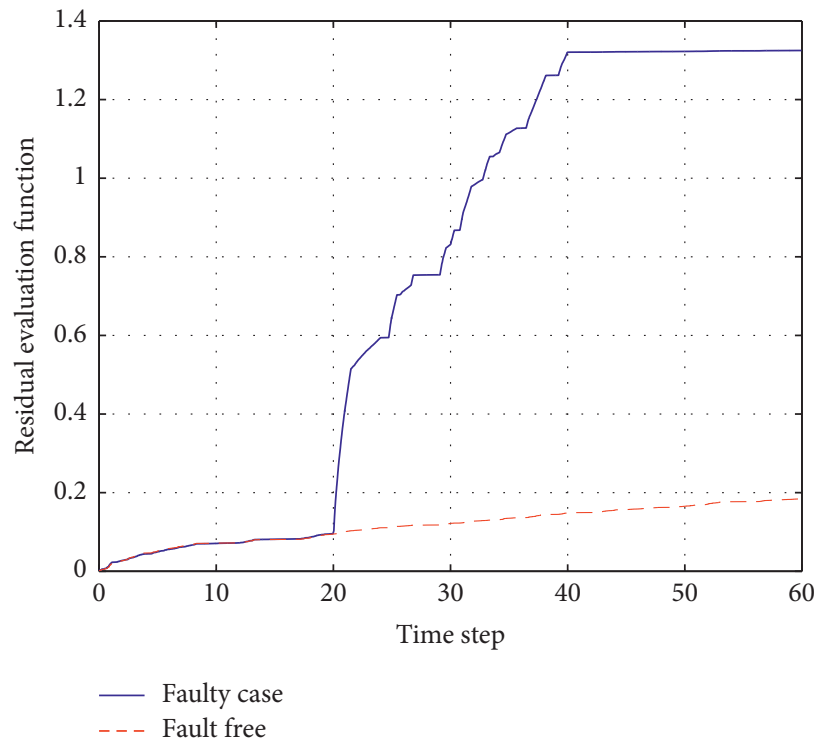

(c)

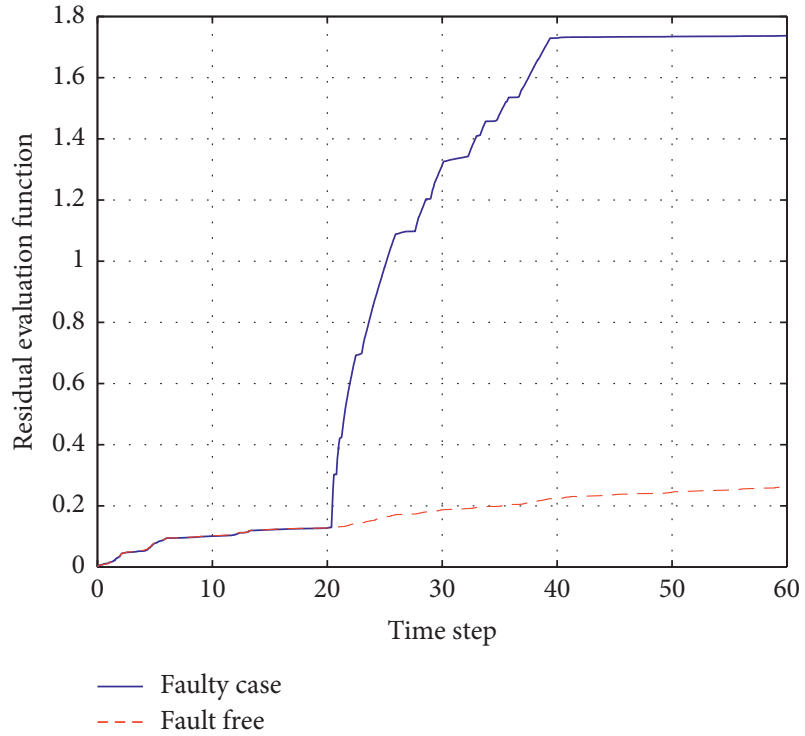

(b)

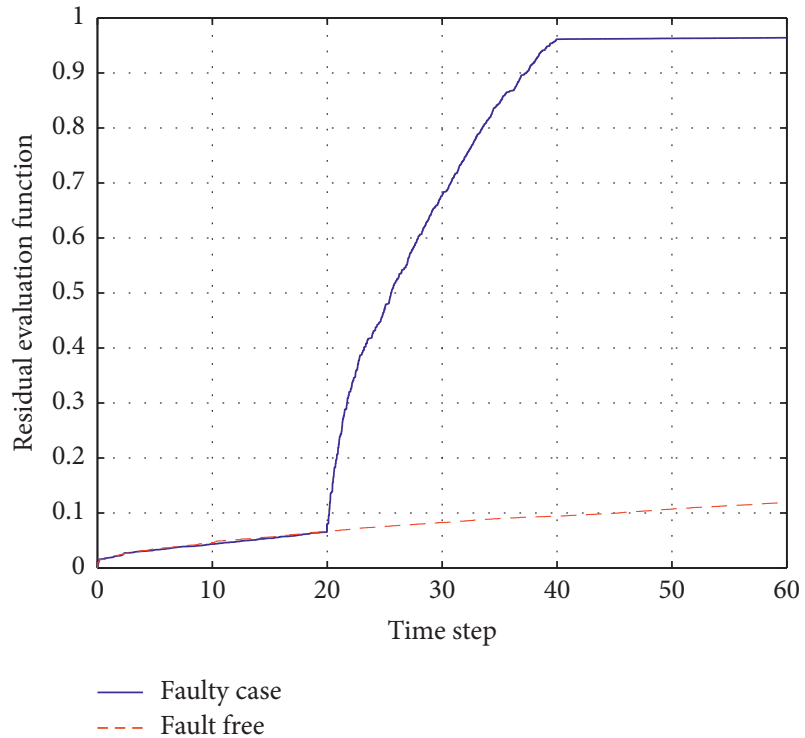

(d)

FIGURE 5: Residual evaluation function curves of reduced-order FDF for four different TRMs cases. (a) Case 1. TRM is completely known. (b) Case 1. TRM is polytopic uncertain. (c) TRM is partly unknown. (d) TRM is completely unknown.

curve of comparison in Figures 3 and 4 that the estimated residual value of the full-order filter is nearer to the weighting fault signal than the estimated residual value of the reduced-order filter. The more the known transition rates are in the TRM, the more accurate the estimated residual values are. Case 2 with polytopic uncertainty TRM is obviously closer to the actual residual value than Case 3 and Case 4. The simulation results further indicate that no matter which case TRM is to be chosen, the designed FDFs are effective and feasible.

Figures 4 and 5 demonstrate the residual evaluation function curves of full-order and reduced-order FDFs for four different TRMs cases, respectively. It can be seen from Figures 4 and 5 that the shortest time step $L_{s_{\min }}$ is close to 20 (s), when $J(\widehat{r}(t))>J_{t h}$, that the weighting fault signal is effective and can improve the performance of the fault detection system. By comparing and analysing residual evaluation function curves of different FDFs and different cases of TRMs, we can draw a conclusion that Case 2 with polytopic uncertain TRM is closer to Case 1 with completely known TRM than Case 3 and Case 4, and the residual evaluation function value of the reduced-order FDF is obviously smaller than that of the full-order FDF and the evaluation time is obviously shortened.

Remark 8. This time (s) represents the shortest time for obtaining $H_{\infty}$ performance level $\gamma_{\min }$, but the value of this 
TABLE 2: Performance comparison results for four different cases of full-order FDFs with time-varying delays.

\begin{tabular}{lcccccc}
\hline \multirow{2}{*}{ TRM } & \multicolumn{2}{c}{$\dot{d}(t)=0.4$} & $t=60$ & \multicolumn{2}{c}{$\dot{d}(t)=0.6$} & $t=60$ \\
& $\gamma_{\min }$ & time $(s)$ & $N_{D V}$ & $\gamma_{\text {min }}$ & time $(s)$ & $N_{D V}$ \\
\hline Case 1 & 0.2946 & 80.0568 & 164 & 0.2980 & 96.0701 & 164 \\
Case 2 & 0.3373 & 137.7648 & 164 & 0.3524 & 144.4304 & 164 \\
Case 3 & 0.4095 & 101.4276 & 164 & 0.4368 & 109.8631 & 164 \\
Case 4 & 0.5749 & 82.8212 & 164 & 0.6078 & 98.6324 & 164 \\
\hline
\end{tabular}

TABLE 3: Performance comparison results for four different cases of reduced-order FDFs with time-varying delays.

\begin{tabular}{lcccccc}
\hline \multirow{2}{*}{ TRM } & \multicolumn{2}{c}{$\dot{d}(t)=0.4$} & $t=60$ & \multicolumn{2}{c}{$\dot{d}(t)=0.6$} & $t=60$ \\
& $\gamma_{\min }$ & time $(s)$ & $N_{D V}$ & $\gamma_{\text {min }}$ & time $(s)$ & $N_{D V}$ \\
\hline Case 1 & 0.2946 & 50.0306 & 136 & 0.2980 & 67.4760 & 136 \\
Case 2 & 0.3373 & 83.2772 & 136 & 0.3524 & 93.1037 & 136 \\
Case 3 & 0.4095 & 70.7835 & 136 & 0.4368 & 82.7555 & 136 \\
Case 4 & 0.5749 & 52.8212 & 136 & 0.6079 & 71.9304 & 136 \\
\hline
\end{tabular}

time is not obtained at one time, by calculating the average value that 50 times of data are obtained on the same computer.

Tables 2 and 3 show the performance comparing fullorder and reduced-order FDFs for the cases of different delay differentials. By comparing Table 2 with Table 3 filters characteristics, we can conclude the following:

(1) Under the condition of the same-order FDFs for different TRMs, the more known transition rates are in the TRM that the $H_{\infty}$ performance level $\gamma_{\min }$ and the conservativeness are less

(2) Under the condition of the same-order FDFs and same TRMs, as the time-varying delays differential value increases, the time to solve $\gamma_{\min }$ significantly increased

(3) Compared with different-order FDFs of the same TRMs, the $H_{\infty}$, performance index $\gamma_{\min }$, and the conservativeness are almost the same, but the number of decision variables $\left(N_{D V}\right)$ reduced-order FDF is obviously less than full-order FDF; moreover, the processing speed of reduced-order FDF is faster than full-order FDF

\section{Conclusions}

In this paper, we propose full-order and reduced-order FDF design methods for CTSMJSs with time-varying delays and polytopic uncertain TRM. Based on the Lyapunov-Krasovskii functional method and convex optimization technique, some delay-dependent sufficient conditions are obtained such that the singular model error augmented system is stochastically admissible with an $H_{\infty}$ performance $\gamma_{\min }$. Finally, two examples are presented to illustrate the effectiveness of the proposed design approach. Moreover, the issue of semi-Markovian jump systems $[42,43]$ is a promising research topic which has a strong practical background. In future, we will study the fault detection of semi-Markovian jump systems.

\section{Data Availability}

No data are used to support this study.

\section{Conflicts of Interest}

The authors declare that there are no conflicts of interest regarding the publication of this paper.

\section{Acknowledgments}

This work was supported by the National Natural Science Foundation of China (61503091) and National Science Foundation of Heilongjiang Province of China (G023016003).

\section{References}

[1] Y. Wei, J. H. Park, H. R. Karimi et al., "Improved stability and stabilization results for stochastic synchronization of continuous-time semi-Markovian jump neural networks with time-varying delay," IEEE Transactions on Neural Networks and Learning Systems, vol. 29, no. 6, pp. 2488-2501, 2017.

[2] Z. Feng and W. X. Zheng, "On reachable set estimation of delay Markovian jump systems with partially known transition probabilities," Journal of the Franklin Institute, vol. 353, no. 15, pp. 3835-3856, 2016.

[3] Y. Wei, J. Qiu, H. R. Karimi, and M. Wang, "Model reduction for continuous-time Markovian jump systems with incomplete statistics of mode information," International Journal of Systems Science, vol. 45, no. 7, pp. 1496-1507, 2014.

[4] L. Zhang, Y. Leng, and P. Colaneri, "Stability and stabilization of discrete-time semi-Markov jump linear systems via semiMarkov kernel approach," IEEE Transactions on Automatic Control, vol. 61, no. 2, pp. 503-508, 2016.

[5] Y. Wei, J. Qiu, and H. R. Karimi, "Reliable output feedback control of discrete-time fuzzy affine systems with actuator faults," IEEE Transactions on Circuits and Systems I: Regular Papers, vol. 64, no. 1, pp. 170-181, 2017.

[6] L. Li, Q. Zhang, and B. Zhu, "Fuzzy stochastic optimal guaranteed cost control of bio-economic singular Markovian jump systems," IEEE Transactions on Cybernetics, vol. 45, no. 11, pp. 2512-2521, 2015.

[7] Y. Wei, J. H. Park, J. Qiu et al., "Reliable output feedback control for piecewise affine systems with Markov-type sensor failure," IEEE Transactions on Circuits and Systems II: Express Briefs, vol. 65, no. 7, pp. 913-917, 2017.

[8] H. Shen, Y. Men, Z.-G. Wu, J. Cao, and G. Lu, "Networkbased quantized control for fuzzy singularly perturbed semiMarkov jump systems and its application," IEEE Transactions on Circuits and Systems I: Regular Papers, vol. 66, no. 3, pp. 1130-1140, 2019.

[9] X. Yao, L. Wu, and W. X. Zheng, "Fault detection filter design for Markovian jump singular systems with intermittent measurements," IEEE Transactions on Signal Processing, vol. 59, no. 7, pp. 3099-3109, 2011.

[10] Z. Feng and J. Lam, "Dissipative control and filtering of discrete-time singular systems," International Journal of Systems Science, vol. 47, no. 11, pp. 2532-2542, 2015. 
[11] S. Xu and J. Lam, "Robust control and filtering of singular systems," 2006.

[12] Z. Feng, J. Lam, S. Xu, and S. Zhou, "Ho control with transients for singular systems," Asian Journal of Control, vol. 18, no. 3, pp. 817-827, 2016.

[13] G. Wang, Q. Zhang, and C. Yang, "Stabilization of singular markovian jump systems with time-varying switching," Elsevier Science Inc, vol. 297, pp. 254-270, 2015.

[14] J. Li, Y. Zhao, Z. Feng, and M. Park, "Reachable set estimation and dissipativity for discrete-time T-S fuzzy singular systems with time-varying delays," Nonlinear Analysis Hybrid Systems, vol. 31, no. 1, pp. 166-179, 2018.

[15] N. K. Kwon, I. S. Park, P. Park, and C. Park, "Dynamic outputfeedback control for singular Markovian jump system: LMI approach," IEEE Transactions on Automatic Control, vol. 62, no. 10, pp. 5396-5400, 2017.

[16] Z. Feng and P. Shi, "Sliding mode control of singular stochastic Markov jump systems," IEEE Transactions on Automatic Control, vol. 62, no. 8, pp. 4266-4273, 2017.

[17] Y. Ma, X. Jia, and D. Liu, "Finite-time dissipative control for singular discrete-Time Markovian jump systems with actuator saturation and partly unknown transition rates," Applied Mathematical Modelling, vol. 53, pp. 49-70, 2018.

[18] Z.-G. Wu, P. Shi, H. Su, and J. Chu, "12-lø filter design for discrete-time singular Markovian jump systems with timevarying delays," Information Sciences, vol. 181, no. 24, pp. 5534-5547, 2011.

[19] Z. Feng and J. Lam, "Reliable dissipative control for singular markovian systems," Asian Journal of Control, vol. 15, no. 3, pp. 901-910, 2013.

[20] J. Qiu, Y. Wei, and H. R. Karimi, "New approach to delaydependent $H_{\infty}$ control for continuous-time Markovian jump systems with time-varying delay and deficient transition descriptions," Journal of the Franklin Institute, vol. 352, no. 1, pp. 189-215, 2015.

[21] H. Li, P. Shi, D. Yao, and L. Wu, "Observer-based adaptive sliding mode control for nonlinear Markovian jump systems," Automatica, vol. 64, pp. 133-142, 2016.

[22] Y. Wei, J. H. Park, J. Qiu, L. Wu, and H. Y. Jung, "Sliding mode control for semi-Markovian jump systems via output feedback," Automatica, vol. 81, pp. 133-141, 2017.

[23] B. Jiang, Y. Kao, H. R. Karimi, and C. Gao, "Stability and stabilization for singular switching semi-Markovian jump systems with generally uncertain transition rates," IEEE Transactions on Automatic Control, vol. 63, no. 11, pp. 3919-3926, 2018.

[24] I. S. Park, N. K. Kwon, and P. Park, "Dynamic outputfeedback control for singular Markovian jump systems with partly unknown transition rates," Nonlinear Dynamics, vol. 95, no. 4, pp. 3149-3160, 2019.

[25] Y. Kao, J. Xie, and C. Wang, "Stabilization of singular markovian jump systems with generally uncertain transition rates," IEEE Transactions on Automatic Control, vol. 59, no. 9, pp. 2604-2610, 2014.

[26] Q. Zhang, L. Li, X.-G. Yan, and S. K. Spurgeon, "Sliding mode control for singular stochastic Markovian jump systems with uncertainties," Automatica, vol. 79, pp. 27-34, 2017.

[27] Y. Wei, J. Qiu, and H. R. Karimi, "Quantized $\mathscr{H} \infty$ filtering for continuous-time markovian jump systems with deficient mode information," Asian Journal of Control, vol. 17, no. 5, pp. 1914-1923, 2015.

[28] H. Gao, T. Chen, and L. Wang, "Robust fault detection with missing measurements," International Journal of Control, vol. 81, no. 5, pp. 804-819, 2008.
[29] I. Hwang, S. Kim, Y. Kim, and C. E. Seah, "A survey of fault detection, isolation, and reconfiguration methods," IEEE Transactions on Control Systems Technology, vol. 18, no. 3, pp. 636-653, 2010.

[30] Y. Wei, J. Qiu, and H. K. Lam, "A novel approach to reliable output feedback control of fuzzy-affine systems with time delays and sensor faults," IEEE Transactions on Fuzzy Systems, vol. 25, no. 6, pp. 1808-1823, 2016.

[31] Y. Wei, J. Qiu, X. Peng, and H.-K. Lam, "T-S fuzzy-affinemodel-based reliable output feedback control of nonlinear systems with actuator faults," Circuits, Systems, and Signal Processing, vol. 37, no. 1, pp. 81-97, 2018.

[32] L. Dai, Singular Control Systems, Springer, New York, NY, USA, 1989.

[33] Z. Feng and P. Shi, "Two equivalent sets: application to singular systems," Automatica, vol. 77, pp. 198-205, 2017.

[34] G. Zhuang, S. Xu, B. Zhang, J. Xia, Y. Chu, and Y. Zou, "Unified filters design for singular Markovian jump systems with time-varying delays," Journal of the Franklin Institute, vol. 353, no. 15, pp. 3739-3768, 2016.

[35] J. Li, Y. Zhao, Z. Feng, and M. Park, "Reachable set estimation and dissipativity for discrete-time T-S fuzzy singular systems with time-varying delays," Nonlinear Analysis: Hybrid Systems, vol. 31, pp. 166-179, 2019.

[36] M. S. Aslam, I. Qaisar, and M. A. Saleem, "Quantized Eventtriggered feedback control under fuzzy system with timevarying delay and Actuator fault," Nonlinear Analysis: Hybrid Systems, vol. 35, pp. 1-14, 2020.

[37] M. S. Aslam and Q. Li, "Quantized dissipative filter design for Markovian switch T-S fuzzy systems with time-varying delays," Soft Computing, vol. 23, no. 21, pp. 11313-11329, 2019.

[38] M. S. Aslam, A. R. Rajput, Q. Li, Y. Liu, and S. A. Niazi, "Adaptive event-triggered mixed $\$ \mathrm{H}_{-}\{\backslash$ infty $\}$ and passivitybased filter design for nonlinear T-S fuzzy markovian switching systems with time-varying delays," IEEE Access, vol. 7, pp. 62577-62591, 2019.

[39] H. R. Karimi and H. Reza, "Robust delay-dependent $\$ \mathrm{H}_{-}$ $\{\backslash$ infty\}\$ control of uncertain time-delay systems with mixed neutral, discrete, and distributed time-delays and markovian switching parameters," IEEE Transactions on Circuits and Systems I: Regular Papers, vol. 58, no. 8, pp. 1910-1923, 2011.

[40] P. Zhang, J. Cao, and G. Wang, "Mode-independent guaranteed cost control of singular Markovian delay jump systems with switching probability rate design," International Journal of Innovative Computing, Information and Control, vol. 10, no. 4, pp. 1291-1303, 2014.

[41] G. Liu, J. H. Park, S. Xu, and G. Zhuang, "Robust non-fragile $\mathrm{H} \infty$ fault detection filter design for delayed singular Markovian jump systems with linear fractional parametric uncertainties," Nonlinear Analysis: Hybrid Systems, vol. 32, pp. 65-78, 2019.

[42] Y. Wei, J. Qiu, H. R. Karimi, and W. Ji, "A novel memory filtering design for semi-Markovian jump time-delay systems," IEEE Transactions on Systems, Man, and Cybernetics: Systems, vol. 48, no. 12, pp. 2229-2241, 2018.

[43] B. Jiang, H. R. Karimi, Y. Kao et al., "Takagi-sugeno model based event-triggered fuzzy sliding mode control of networked control systems with semi-markovian switchings," IEEE Transactions on Fuzzy Systems, vol. 10, 2019. 